\title{
Extensions of Hölder-type inequalities on time scales and their applications
}

\author{
Jing-Feng Tian ${ }^{\mathrm{a}}$, Ming-Hu Ha $\mathrm{H}^{\mathrm{b}, *}$ \\ ${ }^{a}$ College of Science and Technology, North China Electric Power University, Baoding, Hebei Province, 071051, P. R. China. \\ ${ }^{b}$ School of Science, Hebei University of Engineering, Handan, Hebei Province, 056038, P. R. China.
}

Communicated by Sh. Wu

\begin{abstract}
In this paper, we present some new extensions of Hölder-type inequalities on time scales via diamond- $\alpha$ integral. Moreover, the obtained results are used to generalize Minkowski's inequality and Beckenbach-Dresher's inequality on time scales. (C)2017 All rights reserved.

Keywords: Hölder-type inequality, diamond- $\alpha$ integral, time scales, Minkowski's inequality, Beckenbach-Dresher's inequality. 2010 MSC: 26D15, 39A13.
\end{abstract}

\section{Introduction}

Let $f(x)$ and $g(x)$ be continuous real-valued functions on $[a, b]$ and $\frac{1}{p}+\frac{1}{q}=1$.

(I) If $p>1$ and if $f(x) \geqslant 0, g(x) \geqslant 0$, then the following Hölder's inequality holds (see [14]):

$$
\int_{a}^{b} f(x) g(x) d x \leqslant\left(\int_{a}^{b} f^{p}(x) d x\right)^{\frac{1}{p}}\left(\int_{a}^{b} g^{q}(x) d x\right)^{\frac{1}{q}} .
$$

(II) If $0<p<1$ and if $f(x)>0, g(x)>0$, then the following reverse Hölder's inequality (see [11]) holds:

$$
\int_{a}^{b} f(x) g(x) d x \geqslant\left(\int_{a}^{b} f^{p}(x) d x\right)^{\frac{1}{p}}\left(\int_{a}^{b} g^{q}(x) d x\right)^{\frac{1}{q}} .
$$

As is well-known, the above Hölder-type inequalities play an important role in different branches of modern mathematics such as classical real and complex analysis, probability and statistics, numerical analysis, qualitative theory of differential equations and their applications. Various refinements, extensions and applications of inequality (1.1) and (1.2) have appeared in the literature. For example, $\mathrm{Wu}$ $[24,25]$ presented some new sharpened and generalized versions of Hölder's inequality in classical real

\footnotetext{
*Corresponding author

Email addresses: tianjf@ncepu.edu.cn (Jing-Feng Tian), mhhhbu@163.com (Ming-Hu Ha)
} 
analysis. Tian and Ha [20, 21] gave two new properties of generalized Hölder's inequalities. Liu [12] established an important Hölder-type inequality for fuzzy variables. For more detail expositions, the interested reader may consult $[1,2,13,18,19]$, and the references therein. Among various extensions of (1.1) and (1.2), Wong et al. in [23] first established the following time scale versions of Hölder's inequality and reverse Hölder's inequality by using the delta-integral (i.e. $\Delta$-integral).

Theorem 1.1. Let $\mathbb{T}$ be a time scale, $\mathrm{a}, \mathrm{b} \in \mathbb{T}$ with $\mathrm{a}<\mathrm{b}$, let $\frac{1}{\mathrm{p}}+\frac{1}{\mathrm{q}}=1$ and let $\mathrm{f}, \mathrm{g}, \mathrm{h} \in \mathrm{C}_{\mathrm{rd}}\left([\mathrm{a}, \mathrm{b}]_{\mathbb{T}}, \mathbb{R}\right)$. If $p>1$, then

$$
\int_{a}^{b}|h(x) \| f(x) g(x)| \Delta x \leqslant\left(\int_{a}^{b}|h(x) \| f(x)|^{p} \Delta x\right)^{\frac{1}{p}}\left(\int_{a}^{b}|h(x) \| g(x)|^{q} \Delta x\right)^{\frac{1}{q}} .
$$

If $0<p<1$, then the above inequality (1.3) is reversed.

Later in 2008, Özkan et al. [15] presented the following time scale versions of Hölder's inequality and reverse Hölder's inequality by using the nabla-integral (i.e., $\nabla$-integral) and diamond- $\alpha$ integral (i.e. $\diamond_{\alpha}$-integral).

Theorem 1.2. Let $\mathbb{T}$ be a time scale, $\mathrm{a}, \mathrm{b} \in \mathbb{T}$ with $\mathrm{a}<\mathrm{b}$, let $\frac{1}{\mathrm{p}}+\frac{1}{\mathrm{q}}=1$, and let $\mathrm{f}, \mathrm{g}, \mathrm{h} \in \mathrm{C}_{\mathrm{ld}}\left([\mathrm{a}, \mathrm{b}]_{\mathbb{T}}, \mathbb{R}\right)$. If $p>1$, then

$$
\int_{a}^{b}|h(x) \| f(x) g(x)| \nabla x \leqslant\left(\int_{a}^{b}|h(x) \| f(x)|^{p} \nabla x\right)^{\frac{1}{p}}\left(\int_{a}^{b}|h(x) \| g(x)|^{q} \nabla x\right)^{\frac{1}{q}} .
$$

If $0<p<1$, then the above inequality (1.4) is reversed.

Theorem 1.3. Let $\frac{1}{\mathrm{p}}+\frac{1}{\mathrm{q}}=1$, let $\mathbb{T}$ be a time scale, $\mathrm{a}, \mathrm{b} \in \mathbb{T}$ with $\mathrm{a}<\mathrm{b}$, and let $\mathrm{f}, \mathrm{g}, \mathrm{h}:[\mathrm{a}, \mathrm{b}]_{\mathbb{T}} \rightarrow \mathbb{R}$ be $\diamond_{\alpha}$-integrable functions. If $p>1$, then

$$
\int_{a}^{b}|h(x) \| f(x) g(x)| \diamond_{\alpha} x \leqslant\left(\int_{a}^{b}|h(x)||f(x)|^{p} \diamond_{\alpha} x\right)^{\frac{1}{p}}\left(\int_{a}^{b}|h(x) \| g(x)|^{q} \diamond_{\alpha} x\right)^{\frac{1}{q}} .
$$

If $0<\mathrm{p}<1$, then the inequality (1.5) is reversed.

In 2010, Yang [26] derived a functional generalization of diamond- $\alpha$ integral Hölder's inequality on time scales as follows.

Theorem 1.4. Let $\mathbb{T}$ be a time scale, $a, b \in \mathbb{T}$ with $\mathrm{a}<\mathrm{b}$, let $\left\{\mathrm{f}_{\mathrm{j}}(\mathrm{x})\right\}_{j=1}^{\mathrm{m}},\left\{\mathrm{g}_{\mathbf{j}}(\mathrm{x})\right\}_{j=1}^{\mathrm{k}}$ and $\left\{\mathrm{h}_{\mathbf{j}}(\mathrm{x})\right\}_{\mathfrak{j}=1}^{\mathrm{l}}$ be continuous real-valued functions on $[\mathrm{a}, \mathrm{b}]_{\mathbb{T}}$, and let $\mathrm{H}_{\mathrm{l}}\left(\mathrm{x}_{1}, \mathrm{x}_{2}, \cdots, \mathrm{x}_{\mathrm{l}}\right)>0, \mathrm{~F}_{\mathrm{m}}\left(\mathrm{x}_{1}, \mathrm{x}_{2}, \cdots, \mathrm{x}_{\mathrm{m}}\right)$ and $\mathrm{G}_{\mathrm{k}}\left(\mathrm{x}_{1}, \mathrm{x}_{2}, \cdots, \mathrm{x}_{\mathrm{k}}\right)$ be three arbitrary functions of $l, m$ and $k$ variables, respectively. If $p>1$ with $\frac{1}{p}+\frac{1}{q}=1$, then

$$
\begin{gathered}
\int_{a}^{b} H_{l}\left(h_{1}, h_{2}, \ldots, h_{l}\right)\left|F_{m}\left(f_{1}, f_{2}, \ldots, f_{m}\right) G_{k}\left(g_{1}, g_{2}, \ldots, g_{k}\right)\right| \diamond_{\alpha} x \\
\leqslant\left(\int_{a}^{b} H_{l}\left(h_{1}, h_{2}, \ldots, h_{l}\right)\left|F_{m}\left(f_{1}, f_{2}, \ldots, f_{m}\right)\right|^{p} \diamond_{\alpha} x\right)^{1 / p} \\
\quad \times\left(\int_{a}^{b} H_{l}\left(h_{1}, h_{2}, \ldots, h_{l}\right)\left|G_{k}\left(g_{1}, g_{2}, \cdots, g_{k}\right)\right|^{q} \diamond_{\alpha} x\right)^{1 / q} .
\end{gathered}
$$

Recently, Chen and Chen in [6] gave the following reversed version of inequality (1.6).

Theorem 1.5. Let $\mathbb{T}$ be a time scale, $a, b \in \mathbb{T}$ with $\mathrm{a}<\mathrm{b}$, let $\left\{\mathrm{f}_{\mathrm{j}}(\mathrm{x})\right\}_{j=1}^{\mathrm{m}},\left\{\mathrm{g}_{\mathbf{j}}(\mathrm{x})\right\}_{j=1}^{\mathrm{k}}$ and $\left\{\mathrm{h}_{\mathbf{j}}(\mathrm{x})\right\}_{j=1}^{\mathrm{l}}$ be continuous real-valued functions on $[\mathrm{a}, \mathrm{b}]_{\mathbb{T}}$, and let $\mathrm{H}_{\mathrm{l}}\left(\mathrm{x}_{1}, \mathrm{x}_{2}, \cdots, \mathrm{x}_{\mathrm{l}}\right)>0, \mathrm{~F}_{\mathrm{m}}\left(\mathrm{x}_{1}, \mathrm{x}_{2}, \cdots, \mathrm{x}_{\mathrm{m}}\right)$ and $\mathrm{G}_{\mathrm{k}}\left(\mathrm{x}_{1}, \mathrm{x}_{2}, \cdots, \mathrm{x}_{\mathrm{k}}\right)$ be three arbitrary functions of $\mathrm{l}, \mathrm{m}$ and $\mathrm{k}$ variables, respectively. If $0<\mathrm{p}<1, \frac{1}{\mathrm{p}}+\frac{1}{\mathrm{q}}=1$, then

$$
\begin{gathered}
\int_{a}^{b} H_{l}\left(h_{1}, h_{2}, \cdots, h_{l}\right)\left|F_{m}\left(f_{1}, f_{2}, \cdots, f_{m}\right) G_{k}\left(g_{1}, g_{2}, \cdots, g_{k}\right)\right| \diamond_{\alpha} x \\
\geqslant\left(\int_{a}^{b} H_{l}\left(h_{1}, h_{2}, \cdots, h_{l}\right)\left|F_{m}\left(f_{1}, f_{2}, \cdots, f_{m}\right)\right|^{p} \diamond_{\alpha} x\right)^{1 / p} \\
\quad \times\left(\int_{a}^{b} H_{l}\left(h_{1}, h_{2}, \cdots, h_{l}\right)\left|G_{k}\left(g_{1}, g_{2}, \cdots, g_{k}\right)\right|^{q} \diamond_{\alpha} x\right)^{1 / q} .
\end{gathered}
$$


The aim of this paper is to present some new extensions of the above Hölder-type inequalities (1.3), (1.4), (1.5), (1.6), (1.7). Some related inequalities are also considered.

\section{Preliminaries}

In this section, we recall the following concepts related to the notion of time scales. A time scale $\mathbb{T}$ is an arbitrary nonempty closed subset of the real numbers $\mathbb{R}$. The calculus of time scales was initiated by Hilger [8] in order to unify discrete and continuous analysis. The forward jump operator and the backward jump operator [8] are defined by:

$$
\sigma(t):=\inf \{s \in \mathbb{T}: s>t\}, \quad \rho(t):=\sup \{s \in \mathbb{T}: s<t\},
$$

(supplemented by $\inf \emptyset=\sup \mathbb{T}$ and $\sup \emptyset=\inf \mathbb{T}$ ). A point $t \in \mathbb{T}$ is called right-scattered, right-dense, left-scattered, left-dense, if $\sigma(t)>t, \sigma(t)=t, \rho(t)<t, \rho(t)=t$ holds, respectively [8].

Throughout the text, we shall denote a time scales interval by

$$
[a, b]_{\mathbb{T}}=\{t \in \mathbb{T}: a \leqslant t \leqslant b, a, b \in \mathbb{T}\},
$$

and denote

$$
\begin{aligned}
\mathbb{T}^{k} & := \begin{cases}\mathbb{T}-\left\{t_{1}\right\}, & \text { if } \mathbb{T} \text { has a left-scattered maximum } t_{1}, \\
\mathbb{T}, & \text { otherwise, }\end{cases} \\
\mathbb{T}_{k}: & = \begin{cases}\mathbb{T}-\left\{t_{2}\right\}, & \text { if } \mathbb{T} \text { has a right-scattered minimum } t_{2}, \\
\mathbb{T}, & \text { otherwise, }\end{cases}
\end{aligned}
$$

and

$$
\mathbb{T}^{*}=\mathbb{T}^{\mathrm{k}} \cap \mathbb{T}_{\mathrm{k}}
$$

Definition 2.1 ([5]). A function $f: \mathbb{T} \rightarrow \mathbb{R}$ is said to be rd-continuous, if it is continuous at each rightdense point and the left sided limit exists at every left-dense point. The set of all $\mathrm{rd}$-continuous functions is denoted by $\mathrm{C}_{\mathrm{rd}}[\mathbb{T}, \mathbb{R}]$.

Definition 2.2 ([5]). A function $f: \mathbb{T} \rightarrow \mathbb{R}$ is said to be ld-continuous, if it is continuous at all left-dense points in $\mathbb{T}$ and its right-sided limits finite at all right-dense points in $\mathbb{T}$. The set of all ld-continuous functions is denoted by $\mathrm{C}_{\mathrm{ld}}[\mathbb{T}, \mathbb{R}]$.

Definition 2.3 ([9]). A function $f: \mathbb{T}^{k} \rightarrow \mathbb{R}$ is called differentiable at $t \in \mathbb{T}^{k}$, with delta derivative $f^{\Delta}(t)$, if given $\epsilon>0$ there exists a neighborhood $\mathbb{U}$ of $t$ such that

$$
\left|f(\sigma(t))-f(s)-f^{\Delta}(t)(\sigma(t)-s)\right| \leqslant \epsilon|\sigma(t)-s|,
$$

for all $s \in \mathbb{U}$.

Remark 2.4 ([9]). If $\mathbb{T}=\mathbb{R}$, then $\mathrm{f}^{\Delta}(\mathrm{t})$ becomes the usual derivative, that is, $\mathrm{f}^{\Delta}(\mathrm{t})=\mathrm{f}^{\prime}(\mathrm{t})$. If $\mathbb{T}=\mathbb{Z}$, then $f^{\Delta}(t)$ reduces to the usual forward difference, that is, $f^{\Delta}(t)=\Delta f(t)$.

Definition 2.5 ([9]). A function $F: \mathbb{T} \rightarrow \mathbb{R}$ is called a delta antiderivative of $f: \mathbb{T} \rightarrow \mathbb{R}$ provided $F^{\Delta}=f(t)$ holds for all $t \in \mathbb{T}$. In this case we define the delta integral of $f$ by

$$
\int_{s}^{t} f(\tau) \Delta \tau=F(t)-F(s)
$$

where $s, t \in \mathbb{T}$.

Definition 2.6 ([4]). A function $f: \mathbb{T}_{k} \rightarrow \mathbb{R}$ is called nabla differentiable at $t \in \mathbb{T}_{k}$, with nabla derivative $f^{\nabla}(t)$, if given $\epsilon>0$ there exists a neighborhood $\mathbb{V}$ of $t$ such that

$$
\left|f(\rho(t))-f(s)-f^{\nabla}(t)(\rho(t)-s)\right| \leqslant \epsilon|\rho(t)-s|,
$$

for all $s \in \mathbb{V}$. 
Definition 2.7 ([4]). A function $G: \mathbb{T} \rightarrow \mathbb{R}$ is called a nabla antiderivative of $g: \mathbb{T} \rightarrow \mathbb{R}$ provided $\mathrm{G}^{\nabla}=\mathrm{g}(\mathrm{t})$ holds for all $\mathrm{t} \in \mathbb{T}$. In this case we define the nabla integral of $\mathrm{g}$ by

$$
\int_{s}^{t} g(\tau) \nabla \tau=G(t)-G(s)
$$

where $s, t \in \mathbb{T}$.

Definition 2.8 ([17]). Let $\mathbb{T}$ be a time scale and $f(t)$ be differentiable on $\mathbb{T}$ in the $\triangle$ and $\nabla$ senses. For $t \in \mathbb{T}$ we define the diamond- $\alpha$ derivative $f^{\diamond} \alpha(t)$ by

$$
f^{\diamond \alpha}(t)=\alpha f^{\triangle}(t)+(1-\alpha) f^{\nabla}(t), \quad 0 \leqslant \alpha \leqslant 1 .
$$

Thus $f$ is diamond- $\alpha$ differentiable if and only if $f$ is $\Delta$ and $\nabla$ differentiable.

Remark 2.9 ([17]). The diamond- $\alpha$ derivative reduces to the standard $\triangle$ derivative for $\alpha=1$ or the standard $\nabla$ derivative for $\alpha=0$.

Proposition 2.10 ([17]). Let $\mathrm{f}, \mathrm{g}: \mathbb{T} \rightarrow \mathbb{R}$ be diamond- $\alpha$ differentiable at $\mathrm{t} \in \mathbb{T}$. Then

(i) $\mathrm{f}+\mathrm{g}: \mathbb{T} \rightarrow \mathbb{R}$ is diamond- $\alpha$ differentiable at $\mathrm{t} \in \mathbb{T}$ with

$$
(f+g)^{\diamond \alpha}(t)=f^{\diamond \alpha}(t)+g^{\diamond \alpha}(t)
$$

(ii) For any constant $\mathrm{c}, \mathrm{cf}: \mathbb{T} \rightarrow \mathbb{R}$ is diamond- $\alpha$ differentiable at $\mathrm{t} \in \mathbb{T}$ with

$$
(c f)^{\diamond}(t)=c f^{\diamond} \alpha(t) .
$$

(iii) $\mathrm{fg}: \mathbb{T} \rightarrow \mathbb{R}$ is diamond- $\alpha$ differentiable at $\mathrm{t} \in \mathbb{T}$ with

$$
(f g)^{\diamond \alpha}(t)=f^{\diamond_{\alpha}}(t) g(t)+\alpha f^{\sigma}(t) g^{\triangle}(t)+(1-\alpha) f^{\rho}(t) g^{\nabla}(t) .
$$

Definition 2.11 ([17]). Let $a, t \in \mathbb{T}$ and $h: \mathbb{T} \rightarrow \mathbb{R}$. Then the diamond- $\alpha$ integral from $a$ to $t$ of $h$ is defined by

$$
\int_{a}^{t} h(\tau) \diamond_{\alpha} \tau=\alpha \int_{a}^{t} h(\tau) \Delta \tau+(1-\alpha) \int_{a}^{t} h(\tau) \nabla \tau, \quad 0 \leqslant \alpha \leqslant 1
$$

Remark 2.12 ([17]). We may notice that since the $\diamond_{\alpha}$ integral is a combined $\triangle$ and $\nabla$ integral, we in general do not have

$$
\left(\int_{a}^{t} h(\tau) \diamond_{\alpha} \tau\right)^{\diamond_{\alpha}}=h(t), \quad t \in \mathbb{T} .
$$

Proposition 2.13 ([17]). Let $\mathbb{T}$ be a time scale, $\mathrm{a}, \mathrm{b} \in \mathbb{T}$ with $\mathrm{a}<\mathrm{b}$. Assume that $\mathrm{f}(\mathrm{x})$ and $\mathrm{g}(\mathrm{x})$ are $\diamond_{\alpha}$-integrable functions on $[\mathrm{a}, \mathrm{b}]_{\mathbb{T}}$. Then

(i) $\int_{a}^{t}[f(\tau)+g(\tau)] \diamond_{\alpha} \tau=\int_{a}^{t} f(\tau) \diamond_{\alpha} \tau+\int_{a}^{t} g(\tau) \diamond_{\alpha} \tau$;

(ii) $\int_{a}^{t} c f(\tau) \diamond_{\alpha} \tau=c \int_{a}^{t} f(\tau) \diamond_{\alpha} \tau$;

(iii) $\int_{a}^{t} f(\tau) \diamond_{\alpha} \tau=-\int_{t}^{a} f(\tau) \diamond_{\alpha} \tau$;

(iv) $\int_{a}^{t} f(\tau) \diamond_{\alpha} \tau=\int_{a}^{b} f(\tau) \diamond_{\alpha} \tau+\int_{b}^{t} f(\tau) \diamond_{\alpha} \tau$;

(v) $\int_{a}^{a} f(\tau) \diamond_{\alpha} \tau=0$.

For more details on time scales theory, readers may consult $[3,9,10,16,22]$ and the references therein. Now, we present the following extensions of Hölder's and reverse Hölder's inequality on time scales via the diamond- $\alpha$ integral. 


\section{Main results}

In this section, we shall give the following lemmas firstly before we give our results.

Lemma 3.1 ([17]). Let $\mathbb{T}$ be a time scale, $\mathrm{a}, \mathrm{b} \in \mathbb{T}$ with $\mathrm{a}<\mathrm{b}$. Assume that $\mathrm{f}(\mathrm{x})$ and $\mathrm{g}(\mathrm{x})$ are $\diamond_{\alpha}$-integrable functions on $[\mathrm{a}, \mathrm{b}]_{\mathbb{T}}$.

(1) If $f(x) \geqslant 0$ for all $x \in[a, b]_{\mathbb{T}}$, then $\int_{a}^{b} f(x) \diamond_{\alpha} x \geqslant 0$.

(2) If $f(x) \leqslant g(x)$ for all $x \in[a, b]_{\mathbb{T}}$, then $\int_{a}^{b} f(x) \diamond_{\alpha} x \leqslant \int_{a}^{b} g(x) \diamond_{\alpha} x$.

(3) If $\mathrm{f}(\mathrm{x}) \geqslant 0$ for all $\mathrm{x} \in[\mathrm{a}, \mathrm{b}]_{\mathbb{T}}$, then $\mathrm{f}(\mathrm{x})=0$ if and only if $\int_{\mathrm{a}}^{\mathrm{b}} \mathrm{f}(\mathrm{x}) \diamond_{\alpha} \mathrm{x}=0$.

Lemma 3.2 (Radon's inequality, see page 61 in [7]). Let $\zeta_{i}>0$ and $\xi_{i}>0$, for $i=1,2, \cdots, m$.

(a) If $\gamma>0$ or $\gamma<-1$, then

$$
\frac{\left(\sum_{i=1}^{m} \zeta_{i}\right)^{\gamma+1}}{\left(\sum_{i=1}^{m} \xi_{i}\right)^{\gamma}} \leqslant \sum_{i=1}^{m}\left(\frac{\zeta_{i}^{\gamma+1}}{\xi_{i}^{\gamma}}\right) .
$$

(b) If $-1<\gamma<0$, then inequality (3.1) is reversed.

Next, we give the following extension of Schlömilch's inequality [7] on time scales for diamond- $\alpha$ integral.

Lemma 3.3. Let $\mathbb{T}$ be a time scale, $\mathrm{a}, \mathrm{b} \in \mathbb{T}$ with $\mathrm{a}<\mathrm{b}$, let $\left\{\mathrm{f}_{\mathbf{j}}(\mathrm{x})\right\}_{\mathbf{j}=1}^{\mathrm{m}}$ and $\left\{\mathrm{h}_{\mathbf{j}}(\mathrm{x})\right\}_{\mathbf{j}=1}^{\mathrm{l}}$ be continuous real-valued functions on $[\mathrm{a}, \mathrm{b}]_{\mathbb{T}}$, let $\mathrm{H}_{\mathrm{l}}\left(\mathrm{x}_{1}, \mathrm{x}_{2}, \cdots, \mathrm{x}_{\mathrm{l}}\right)>0$ with $\int_{\mathrm{a}}^{\mathrm{b}} \mathrm{H}_{\mathrm{l}}\left(\mathrm{h}_{1}, \mathrm{~h}_{2}, \cdots, \mathrm{h}_{\mathrm{l}}\right) \diamond_{\alpha} \mathrm{x}=1$ and let $\mathrm{F}_{\mathrm{m}}\left(\mathrm{x}_{1}, \mathrm{x}_{2}, \cdots, \mathrm{x}_{\mathrm{m}}\right)$ be an arbitrary function of $\mathrm{m}$ variables. If $\mathrm{s}>\mathrm{r}>0$, then

$$
\begin{aligned}
& \left(\int_{a}^{b} H_{l}\left(h_{1}, h_{2}, \cdots, h_{l}\right)\left|F_{m}\left(f_{1}, f_{2}, \cdots, f_{m}\right)\right|^{s} \diamond_{\alpha} x\right)^{\frac{1}{s}} \\
& \quad \geqslant\left(\int_{a}^{b} H_{l}\left(h_{1}, h_{2}, \cdots, h_{l}\right)\left|F_{m}\left(f_{1}, f_{2}, \cdots, f_{m}\right)\right|^{r} \diamond_{\alpha} x\right)^{\frac{1}{r}} .
\end{aligned}
$$

Proof. In order to prove Lemma 3.3, we need Bernoulli's inequality, that is, if $x>0$, then

$$
x^{p} \geqslant p x+1-p, \quad \text { if } p>1 .
$$

Since $s>r>0$, we have $\frac{s}{r}>1$. Thus, by Bernoulli's inequality, we have

$$
\begin{aligned}
& \int_{a}^{b} H_{l}\left(h_{1}, h_{2}, \cdots, h_{l}\right)\left(\frac{\left|F_{m}\left(f_{1}, f_{2}, \cdots, f_{m}\right)\right|}{\int_{a}^{b} H_{l}\left(h_{1}, h_{2}, \cdots, h_{l}\right)\left|F_{m}\left(f_{1}, f_{2}, \cdots, f_{m}\right)\right| \diamond_{\alpha} x}\right)^{\frac{s}{r}} \diamond_{\alpha} x \\
& \quad \geqslant \int_{a}^{b} H_{l}\left(h_{1}, h_{2}, \cdots, h_{l}\right)\left(\frac{\frac{s}{r}\left|F_{m}\left(f_{1}, f_{2}, \cdots, f_{m}\right)\right|}{\int_{a}^{b} H_{l}\left(h_{1}, h_{2}, \cdots, h_{l}\right)\left|F_{m}\left(f_{1}, f_{2}, \cdots, f_{m}\right)\right| \diamond_{\alpha} x}+1-\frac{s}{r}\right) \diamond_{\alpha} x \\
& \quad=1,
\end{aligned}
$$

that is,

$$
\begin{aligned}
& \int_{a}^{b} H_{l}\left(h_{1}, h_{2}, \cdots, h_{l}\right)\left|F_{m}\left(f_{1}, f_{2}, \cdots, f_{m}\right)\right|^{\frac{s}{r}} \diamond_{\alpha} x \\
& \quad \geqslant\left(\int_{a}^{b} H_{l}\left(h_{1}, h_{2}, \cdots, h_{l}\right)\left|F_{m}\left(f_{1}, f_{2}, \cdots, f_{m}\right)\right| \diamond_{\alpha} x\right)^{\frac{s}{r}} .
\end{aligned}
$$


Letting $F_{m}$ be replaced by $F_{m}^{r}$ in the above inequality, we get

$$
\begin{aligned}
& \int_{a}^{b} H_{l}\left(h_{1}, h_{2}, \cdots, h_{l}\right)\left|F_{m}\left(f_{1}, f_{2}, \cdots, f_{m}\right)\right|^{s} \diamond_{\alpha} x \\
& \quad \geqslant\left(\int_{a}^{b} H_{l}\left(h_{1}, h_{2}, \cdots, h_{l}\right)\left|F_{m}\left(f_{1}, f_{2}, \cdots, f_{m}\right)\right|^{r} \diamond_{\alpha} x\right)^{\frac{s}{r}} .
\end{aligned}
$$

Hence

$$
\begin{aligned}
& \left(\int_{a}^{b} H_{l}\left(h_{1}, h_{2}, \cdots, h_{l}\right)\left|F_{m}\left(f_{1}, f_{2}, \cdots, f_{m}\right)\right|^{s} \diamond_{\alpha} x\right)^{\frac{1}{s}} \\
& \quad \geqslant\left(\int_{a}^{b} H_{l}\left(h_{1}, h_{2}, \cdots, h_{l}\right)\left|F_{m}\left(f_{1}, f_{2}, \cdots, f_{m}\right)\right|^{r} \diamond_{\alpha} x\right)^{\frac{1}{r}} .
\end{aligned}
$$

Thus, the proof of Lemma 3.3 is completed.

By the same method as in Lemma 3.3, we can obtain the following Schlömilch's inequality [7] on time scales.

Lemma 3.4. Let $\mathbb{T}$ be a time scale, $\mathrm{a}, \mathrm{b} \in \mathbb{T}$ with $\mathrm{a}<\mathrm{b}$, let $\mathrm{h}(\mathrm{x}):[\mathrm{a}, \mathrm{b}]_{\mathbb{T}} \rightarrow[0,+\infty)$ be $\diamond_{\alpha}$-integrable function such that $\int_{\mathrm{a}}^{\mathrm{b}} \mathrm{h}(\mathrm{x}) \diamond_{\alpha} \mathrm{x}=1$, and let $\mathrm{f}(\mathrm{x}):[\mathrm{a}, \mathrm{b}]_{\mathbb{T}} \rightarrow[0,+\infty)$ be $\diamond_{\alpha}$-integrable function. If $\mathrm{s}>\mathrm{r}>0$, then

$$
\left(\int_{a}^{b} h(x) f^{s}(x) \diamond_{\alpha} x\right)^{\frac{1}{s}} \geqslant\left(\int_{a}^{b} h(x) f^{r}(x) \diamond_{\alpha} x\right)^{\frac{1}{r}} .
$$

Now, we present the following $\diamond \alpha$-integrable Hölder-type inequalities.

Theorem 3.5. Let $\frac{1}{p}+\frac{1}{q}=\frac{1}{r}$ with $p, q, r \in \mathbb{R}-\{0\}$, let $\mathbb{T}$ be a time scale, $a, b \in \mathbb{T}$ with $a<b$, let $\left\{\mathrm{f}_{\mathfrak{j}}(\mathrm{x})\right\}_{\mathbf{j}=1}^{\mathrm{m}},\left\{\mathrm{g}_{\mathbf{j}}(\mathrm{x})\right\}_{\mathbf{j}=1}^{\mathrm{k}}$ and $\left\{\mathrm{h}_{\mathbf{j}}(\mathrm{x})\right\}_{\mathbf{j}=1}^{\mathrm{l}}$ be continuous real-valued functions on $[\mathrm{a}, \mathrm{b}]_{\mathbb{T}}$ and let $\mathrm{H}_{\mathrm{l}}\left(\mathrm{x}_{1}, \mathrm{x}_{2}, \cdots, \mathrm{x}_{\mathrm{l}}\right)>$ $0, \mathrm{~F}_{\mathrm{m}}\left(\mathrm{x}_{1}, \mathrm{x}_{2}, \cdots, \mathrm{x}_{\mathrm{m}}\right)$ and $\mathrm{G}_{\mathrm{k}}\left(\mathrm{x}_{1}, \mathrm{x}_{2}, \cdots, \mathrm{x}_{\mathrm{k}}\right)$ be three arbitrary functions of $\mathrm{l}, \mathrm{m}$ and $\mathrm{k}$ variables, respectively.

(I) If $\mathrm{p}>0$ and $\mathrm{q}>0$ or $\mathrm{p}>0, \mathrm{q}<0$ and $\mathrm{r}<0$, then

$$
\begin{gathered}
\left(\int_{a}^{b} H_{l}\left(h_{1}, h_{2}, \cdots, h_{l}\right)\left|F_{m}^{r}\left(f_{1}, f_{2}, \cdots, f_{m}\right) G_{k}^{r}\left(g_{1}, g_{2}, \cdots, g_{k}\right)\right| \diamond_{\alpha} x\right)^{\frac{1}{r}} \\
\leqslant\left(\int_{a}^{b} H_{l}\left(h_{1}, h_{2}, \cdots, h_{l}\right)\left|F_{m}\left(f_{1}, f_{2}, \cdots, f_{m}\right)\right|^{p} \diamond_{\alpha} x\right)^{\frac{1}{p}} \\
\quad \times\left(\int_{a}^{b} H_{l}\left(h_{1}, h_{2}, \cdots, h_{l}\right)\left|G_{k}\left(g_{1}, g_{2}, \cdots, g_{k}\right)\right|^{q} \diamond_{\alpha} x\right)^{\frac{1}{q}} .
\end{gathered}
$$

(II) If $\mathrm{p}>0, \mathrm{q}<0$ and $\mathrm{r}>0$ or $\mathrm{p}<0$ and $\mathrm{q}<0$, then

$$
\begin{gathered}
\left(\int_{a}^{b} H_{l}\left(h_{1}, h_{2}, \cdots, h_{l}\right)\left|F_{m}^{r}\left(f_{1}, f_{2}, \cdots, f_{m}\right) G_{k}^{r}\left(g_{1}, g_{2}, \cdots, g_{k}\right)\right| \diamond_{\alpha} x\right)^{\frac{1}{r}} \\
\geqslant\left(\int_{a}^{b} H_{l}\left(h_{1}, h_{2}, \cdots, h_{l}\right)\left|F_{m}\left(f_{1}, f_{2}, \cdots, f_{m}\right)\right|^{p} \diamond_{\alpha} x\right)^{\frac{1}{p}} \\
\quad \times\left(\int_{a}^{b} H_{l}\left(h_{1}, h_{2}, \cdots, h_{l}\right)\left|G_{k}\left(g_{1}, g_{2}, \cdots, g_{k}\right)\right|^{q} \diamond_{\alpha} x\right)^{\frac{1}{q}} .
\end{gathered}
$$

Proof.

(I). Case 1: Let $p>0, q>0$. From $\frac{1}{p}+\frac{1}{q}=\frac{1}{r}$, we find that

$$
\frac{p}{r}>1, \frac{1}{p / r}+\frac{1}{q / r}=1 \text {. }
$$

Hence, from inequality (1.6) we have 


$$
\begin{aligned}
& \int_{a}^{b} H_{l}\left(h_{1}, h_{2}, \cdots, h_{l}\right)\left|F_{m}^{r}\left(f_{1}, f_{2}, \cdots, f_{m}\right) G_{k}^{r}\left(g_{1}, g_{2}, \cdots, g_{k}\right)\right| \diamond_{\alpha} x \\
& \leqslant\left(\int_{a}^{b} H_{l}\left(h_{1}, h_{2}, \cdots, h_{l}\right)\left|F_{m}^{r}\left(f_{1}, f_{2}, \cdots, f_{m}\right)\right|^{\frac{p}{r}} \diamond_{\alpha} x\right)^{\frac{r}{p}} \\
& \quad \times\left(\int_{a}^{b} H_{l}\left(h_{1}, h_{2}, \cdots, h_{l}\right)\left|G_{k}^{r}\left(g_{1}, g_{2}, \cdots, g_{k}\right)\right|^{\frac{q}{r}} \diamond_{\alpha} x\right)^{\frac{r}{q}},
\end{aligned}
$$

which implies

$$
\begin{gathered}
\left(\int_{a}^{b} H_{l}\left(h_{1}, h_{2}, \cdots, h_{l}\right)\left|F_{m}^{r}\left(f_{1}, f_{2}, \cdots, f_{m}\right) G_{k}^{r}\left(g_{1}, g_{2}, \cdots, g_{k}\right)\right| \diamond_{\alpha} x\right)^{\frac{1}{r}} \\
\leqslant\left(\int_{a}^{b} H_{l}\left(h_{1}, h_{2}, \cdots, h_{l}\right)\left|F_{m}\left(f_{1}, f_{2}, \cdots, f_{m}\right)\right|^{p} \diamond_{\alpha} x\right)^{\frac{1}{p}} \\
\quad \times\left(\int_{a}^{b} H_{l}\left(h_{1}, h_{2}, \cdots, h_{l}\right)\left|G_{k}\left(g_{1}, g_{2}, \cdots, g_{k}\right)\right|^{q} \diamond_{\alpha} x\right)^{\frac{1}{q}} .
\end{gathered}
$$

Case 2: Let $p>0, q<0$ and $r<0$. By the same method as in the above Case 1, we can obtain the desired inequality (3.2).

(II). Case 3: Let $p>0, q<0$ and $r>0$. From $\frac{1}{p}+\frac{1}{q}=\frac{1}{r}$, we find that

$$
\frac{r}{p}>1, \frac{1}{r / p}+\frac{1}{-q / p}=\frac{p}{r}-\frac{p}{q}=p\left(\frac{1}{r}-\frac{1}{q}\right)=1 .
$$

Thus, from inequality (1.6) we have

$$
\begin{aligned}
\int_{a}^{b} & H_{l}\left(h_{1}, h_{2}, \cdots, h_{l}\right)\left|F_{m}^{p}\left(f_{1}, f_{2}, \cdots, f_{m}\right)\right| \diamond_{\alpha} x \\
= & \int_{a}^{b} H_{l}\left(h_{1}, h_{2}, \cdots, h_{l}\right)\left|F_{m}^{p}\left(f_{1}, f_{2}, \cdots, f_{m}\right)\right|\left|G_{k}^{p}\left(g_{1}, g_{2}, \cdots, g_{k}\right)\right|\left|G_{k}^{-p}\left(g_{1}, g_{2}, \cdots, g_{k}\right)\right| \diamond_{\alpha} x \\
\leqslant & \left(\int_{a}^{b} H_{l}\left(h_{1}, h_{2}, \cdots, h_{l}\right)\left|F_{m}^{p}\left(f_{1}, f_{2}, \cdots, f_{m}\right) G_{k}^{p}\left(g_{1}, g_{2}, \cdots, g_{k}\right)\right|^{\frac{r}{p}} \diamond_{\alpha} x\right)^{\frac{p}{r}} \\
& \times\left(\int_{a}^{b} H_{l}\left(h_{1}, h_{2}, \cdots, h_{l}\right)\left|G_{k}^{-p}\left(g_{1}, g_{2}, \cdots, g_{k}\right)\right|^{-\frac{q}{p}} \diamond_{\alpha} x\right)^{-\frac{p}{q}} \\
= & \left(\int_{a}^{b} H_{l}\left(h_{1}, h_{2}, \cdots, h_{l}\right)\left|F_{m}^{r}\left(f_{1}, f_{2}, \cdots, f_{m}\right) G_{k}^{r}\left(g_{1}, g_{2}, \cdots, g_{k}\right)\right| \diamond_{\alpha} x\right)^{\frac{p}{r}} \\
& \times\left(\int_{a}^{b} H_{l}\left(h_{1}, h_{2}, \cdots, h_{l}\right)\left|G_{k}^{q}\left(g_{1}, g_{2}, \cdots, g_{k}\right)\right| \diamond_{\alpha} x\right)^{-\frac{p}{q}},
\end{aligned}
$$

which implies

$$
\begin{aligned}
& \left(\int_{a}^{b} H_{l}\left(h_{1}, h_{2}, \cdots, h_{l}\right)\left|F_{m}^{p}\left(f_{1}, f_{2}, \ldots, f_{m}\right)\right| \diamond_{\alpha} x\right)^{\frac{1}{p}} \\
& \leqslant\left(\int_{a}^{b} H_{l}\left(h_{1}, h_{2}, \ldots, h_{l}\right)\left|F_{m}^{r}\left(f_{1}, f_{2}, \cdots, f_{m}\right) G_{k}^{r}\left(g_{1}, g_{2}, \cdots, g_{k}\right)\right| \diamond_{\alpha} x\right)^{\frac{1}{r}} \\
& \quad \times\left(\int_{a}^{b} H_{l}\left(h_{1}, h_{2}, \cdots, h_{l}\right)\left|G_{k}^{q}\left(g_{1}, g_{2}, \cdots, g_{k}\right)\right| \diamond_{\alpha} x\right)^{-\frac{1}{q}} .
\end{aligned}
$$


Therefore, by inequality (3.4), we have

$$
\begin{gathered}
\left(\int_{a}^{b} H_{l}\left(h_{1}, h_{2}, \cdots, h_{l}\right)\left|F_{m}^{r}\left(f_{1}, f_{2}, \cdots, f_{m}\right) G_{k}^{r}\left(g_{1}, g_{2}, \cdots, g_{k}\right)\right| \diamond_{\alpha} x\right)^{\frac{1}{r}} \\
\geqslant\left(\int_{a}^{b} H_{l}\left(h_{1}, h_{2}, \cdots, h_{l}\right)\left|F_{m}^{p}\left(f_{1}, f_{2}, \cdots, f_{m}\right)\right| \diamond_{\alpha} x\right)^{\frac{1}{p}} \\
\quad \times\left(\int_{a}^{b} H_{l}\left(h_{1}, h_{2}, \cdots, h_{l}\right)\left|G_{k}^{q}\left(g_{1}, g_{2}, \cdots, g_{k}\right)\right| \diamond_{\alpha} x\right)^{\frac{1}{q}} .
\end{gathered}
$$

Case 4: Let $p<0$ and $q<0$. By the same method as in the above Case 3, we can obtain the desired inequality (3.3). The proof of Theorem 3.5 is completed.

Corollary 3.6. $(\mathbb{T}=\mathbb{R})$ Let $\frac{1}{p}+\frac{1}{q}=\frac{1}{r}$ with $p, q, r \in \mathbb{R}-\{0\}$, let $\left\{f_{j}(x)\right\}_{j=1}^{m},\left\{g_{j}(x)\right\}_{j=1}^{k}$ and $\left\{h_{j}(x)\right\}_{j=1}^{l}$ be continuous real-valued functions on $[\mathrm{a}, \mathrm{b}]$ and let $\mathrm{H}_{\mathrm{l}}\left(\mathrm{x}_{1}, \mathrm{x}_{2}, \cdots, \mathrm{x}_{\mathrm{l}}\right)>0, \mathrm{~F}_{\mathrm{m}}\left(\mathrm{x}_{1}, \mathrm{x}_{2}, \cdots, \mathrm{x}_{\mathrm{m}}\right)$ and $\mathrm{G}_{\mathrm{k}}\left(\mathrm{x}_{1}, \mathrm{x}_{2}, \cdots, \mathrm{x}_{\mathrm{k}}\right)$ be three arbitrary functions of $\mathrm{l}, \mathrm{m}$ and $\mathrm{k}$ variables, respectively.

(I) If $\mathrm{p}>0$ and $\mathrm{q}>0$ or $\mathrm{p}>0$, $\mathrm{q}<0$ and $\mathrm{r}<0$, then

$$
\begin{gathered}
\left(\int_{a}^{b} H_{l}\left(h_{1}, h_{2}, \cdots, h_{l}\right)\left|F_{m}^{r}\left(f_{1}, f_{2}, \cdots, f_{m}\right) G_{k}^{r}\left(g_{1}, g_{2}, \cdots, g_{k}\right)\right| d x\right)^{\frac{1}{r}} \\
\leqslant\left(\int_{a}^{b} H_{l}\left(h_{1}, h_{2}, \cdots, h_{l}\right)\left|F_{m}\left(f_{1}, f_{2}, \cdots, f_{m}\right)\right|^{p} d x\right)^{\frac{1}{p}} \\
\quad \times\left(\int_{a}^{b} H_{l}\left(h_{1}, h_{2}, \cdots, h_{l}\right)\left|G_{k}\left(g_{1}, g_{2}, \cdots, g_{k}\right)\right|^{q} d x\right)^{\frac{1}{q}} .
\end{gathered}
$$

(II) If $\mathrm{p}>0, \mathrm{q}<0$ and $\mathrm{r}>0$ or $\mathrm{p}<0$ and $\mathrm{q}<0$, then

$$
\begin{gathered}
\left(\int_{a}^{b} H_{l}\left(h_{1}, h_{2}, \cdots, h_{l}\right)\left|F_{m}^{r}\left(f_{1}, f_{2}, \cdots, f_{m}\right) G_{k}^{r}\left(g_{1}, g_{2}, \cdots, g_{k}\right)\right| d x\right)^{\frac{1}{r}} \\
\geqslant\left(\int_{a}^{b} H_{l}\left(h_{1}, h_{2}, \cdots, h_{l}\right)\left|F_{m}\left(f_{1}, f_{2}, \cdots, f_{m}\right)\right|^{p} d x\right)^{\frac{1}{p}} \\
\quad \times\left(\int_{a}^{b} H_{l}\left(h_{1}, h_{2}, \cdots, h_{l}\right)\left|G_{k}\left(g_{1}, g_{2}, \cdots, g_{k}\right)\right|^{q} d x\right)^{\frac{1}{q}} .
\end{gathered}
$$

Corollary 3.7. $(\mathbb{T}=\mathbb{Z})$ Let $\frac{1}{p}+\frac{1}{q}=\frac{1}{r}$ with $p, q, r \in \mathbb{R}-\{0\}$, let $\left\{a_{i 1}, a_{i 2}, \ldots, a_{i m}\right\}_{i=1}^{n},\left\{b_{i 1}, b_{i 2}, \ldots, b_{i k}\right\}_{i=1}^{n}$ and $\left\{c_{i 1}, c_{i 2}, \ldots, c_{i l}\right\}_{i=1}^{n}$ be real numbers for any $m, k, l \in \mathbb{N}$, and let $H_{l}\left(x_{1}, x_{2}, \cdots, x_{l}\right)>0, F_{m}\left(x_{1}, x_{2}, \cdots, x_{m}\right)$ and $\mathrm{G}_{\mathrm{k}}\left(\mathrm{x}_{1}, \mathrm{x}_{2}, \cdots, \mathrm{x}_{\mathrm{k}}\right)$ be three arbitrary functions of $\mathrm{l}, \mathrm{m}$ and $\mathrm{k}$ variables, respectively.

(I) If $\mathrm{p}>0$ and $\mathrm{q}>0$ or $\mathrm{p}>0, \mathrm{q}<0$ and $\mathrm{r}<0$, then

$$
\begin{gathered}
\left(\sum_{i=1}^{n} H_{l}\left(c_{i 1}, c_{i 2}, \cdots, c_{i l}\right)\left|F_{m}^{r}\left(a_{i 1}, a_{i 2}, \cdots, a_{i m}\right) G_{k}^{r}\left(b_{i 1}, b_{i 2}, \cdots, b_{i k}\right)\right|\right)^{\frac{1}{r}} \\
\leqslant\left(\sum_{i=1}^{n} H_{l}\left(c_{i 1}, c_{i 2}, \cdots, c_{i l}\right)\left|F_{m}\left(a_{i 1}, a_{i 2}, \cdots, a_{i m}\right)\right|^{p}\right)^{\frac{1}{p}} \\
\quad \times\left(\sum_{i=1}^{n} H_{l}\left(c_{i 1}, c_{i 2}, \cdots, c_{i l}\right)\left|G_{k}\left(b_{i 1}, b_{i 2}, \cdots, b_{i k}\right)\right|^{q}\right)^{\frac{1}{q}} .
\end{gathered}
$$


(II) If $\mathrm{p}>0, \mathrm{q}<0$ and $\mathrm{r}>0$ or $\mathrm{p}<0$ and $\mathrm{q}<0$, then

$$
\begin{gathered}
\left(\sum_{i=1}^{n} H_{l}\left(c_{i 1}, c_{i 2}, \cdots, c_{i l}\right)\left|F_{m}^{r}\left(a_{i 1}, a_{i 2}, \cdots, a_{i m}\right) G_{k}^{r}\left(b_{i 1}, b_{i 2}, \cdots, b_{i k}\right)\right|\right)^{\frac{1}{r}} \\
\geqslant\left(\sum_{i=1}^{n} H_{l}\left(c_{i 1}, c_{i 2}, \cdots, c_{i l}\right)\left|F_{m}\left(a_{i 1}, a_{i 2}, \cdots, a_{i m}\right)\right|^{p}\right)^{\frac{1}{p}} \\
\quad \times\left(\sum_{i=1}^{n} H_{l}\left(c_{i 1}, c_{i 2}, \cdots, c_{i l}\right)\left|G_{k}\left(b_{i 1}, b_{i 2}, \cdots, b_{i k}\right)\right|^{q}\right)^{\frac{1}{q}} .
\end{gathered}
$$

Theorem 3.8. Let $\mathbb{T}$ be a time scale, $a, b \in \mathbb{T}$ with $\mathrm{a}<\mathrm{b}$, let $\left\{\mathrm{f}_{\mathbf{j}}(\mathrm{x})\right\}_{\mathbf{j}=1}^{\mathrm{m}},\left\{\boldsymbol{g}_{\mathbf{j}}(\mathrm{x})\right\}_{\mathbf{j}=1}^{\mathrm{k}}$ and $\left\{\mathrm{h}_{\mathbf{j}}(\mathrm{x})\right\}_{\mathbf{j}=1}^{\mathrm{l}}$ be continuous real-valued functions on $[\mathrm{a}, \mathrm{b}]_{\mathbb{T}}$, let $\mathrm{H}_{\mathrm{l}}\left(\mathrm{x}_{1}, \mathrm{x}_{2}, \cdots, \mathrm{x}_{\mathrm{l}}\right)>0$ with $\int_{\mathrm{a}}^{\mathrm{b}} \mathrm{H}_{\mathrm{l}}\left(\mathrm{h}_{1}, \mathrm{~h}_{2}, \cdots, \mathrm{h}_{\mathrm{l}}\right) \diamond_{\alpha} \mathrm{x}=1$ and let $\mathrm{F}_{\mathrm{m}}\left(\mathrm{x}_{1}, \mathrm{x}_{2}, \cdots, \mathrm{x}_{\mathrm{m}}\right)$ and $\mathrm{G}_{\mathrm{k}}\left(\mathrm{x}_{1}, \mathrm{x}_{2}, \cdots, \mathrm{x}_{\mathrm{k}}\right)$ be two arbitrary functions of $\mathrm{m}$ and $\mathrm{k}$ variables, respectively. If $\mathrm{p}>0$, $\mathrm{q}>0$ with $\frac{1}{\mathrm{p}}+\frac{1}{\mathrm{q}}<1$, then

$$
\begin{aligned}
& \int_{a}^{b} H_{l}\left(h_{1}, h_{2}, \cdots, h_{l}\right)\left|F_{m}\left(f_{1}, f_{2}, \cdots, f_{m}\right) G_{k}\left(g_{1}, g_{2}, \cdots, g_{k}\right)\right| \diamond_{\alpha} x \\
& \leqslant\left(\int_{a}^{b} H_{l}\left(h_{1}, h_{2}, \cdots, h_{l}\right)\left|F_{m}\left(f_{1}, f_{2}, \cdots, f_{m}\right)\right|^{p} \diamond_{\alpha} x\right)^{\frac{1}{p}} \\
& \quad \times\left(\int_{a}^{b} H_{l}\left(h_{1}, h_{2}, \cdots, h_{l}\right)\left|G_{k}\left(g_{1}, g_{2}, \cdots, g_{k}\right)\right|^{q} \diamond_{\alpha} x\right)^{\frac{1}{q}} .
\end{aligned}
$$

Proof. Write $\rho:=\frac{1}{p}+\frac{1}{q}, \alpha=\rho p, \beta=q \rho$. Then $\alpha>1, \beta>1$ and $\frac{1}{\alpha}+\frac{1}{\beta}=1$. Hence, from Lemma 3.3 with $\alpha<p, \beta<q$ and Theorem 1.4, we find that

$$
\begin{aligned}
\int_{a}^{b} H_{l}\left(h_{1}, h_{2}, \cdots, h_{l}\right)\left|F_{m}\left(f_{1}, f_{2}, \cdots, f_{m}\right) G_{k}\left(g_{1}, g_{2}, \cdots, g_{k}\right)\right| \diamond_{\alpha} x \\
\leqslant\left(\int_{a}^{b} H_{l}\left(h_{1}, h_{2}, \cdots, h_{l}\right)\left|F_{m}\left(f_{1}, f_{2}, \cdots, f_{m}\right)\right|^{\alpha} \diamond_{\alpha} x\right)^{\frac{1}{\alpha}} \\
\quad \times\left(\int_{a}^{b} H_{l}\left(h_{1}, h_{2}, \cdots, h_{l}\right)\left|G_{k}\left(g_{1}, g_{2}, \cdots, g_{k}\right)\right|^{\beta} \diamond_{\alpha} x\right)^{\frac{1}{\beta}} \\
\leqslant\left(\int_{a}^{b} H_{l}\left(h_{1}, h_{2}, \cdots, h_{l}\right)\left|F_{m}\left(f_{1}, f_{2}, \cdots, f_{m}\right)\right|^{p} \diamond_{\alpha} x\right)^{\frac{1}{p}} \\
\quad \times\left(\int_{a}^{b} H_{l}\left(h_{1}, h_{2}, \cdots, h_{l}\right)\left|G_{k}\left(g_{1}, g_{2}, \cdots, g_{k}\right)\right|^{q} \diamond_{\alpha} x\right)^{\frac{1}{q}} .
\end{aligned}
$$

The proof of Theorem 3.8 is completed.

Corollary 3.9. $(\mathbb{T}=\mathbb{R})$ Let $\left\{f_{j}(x)\right\}_{j=1}^{m},\left\{g_{j}(x)\right\}_{j=1}^{k}$ and $\left\{h_{j}(x)\right\}_{j=1}^{l}$ be continuous real-valued functions on $[a, b]$, let $\mathrm{H}_{\mathrm{l}}\left(\mathrm{x}_{1}, \mathrm{x}_{2}, \cdots, \mathrm{x}_{\mathrm{l}}\right)>0$ with $\int_{\mathrm{a}}^{\mathrm{b}} \mathrm{H}_{\mathrm{l}}\left(\mathrm{h}_{1}, \mathrm{~h}_{2}, \cdots, \mathrm{h}_{\mathrm{l}}\right) \mathrm{dx}=1$ and let $\mathrm{F}_{\mathrm{m}}\left(\mathrm{x}_{1}, \mathrm{x}_{2}, \cdots, \mathrm{x}_{\mathrm{m}}\right)$ and $\mathrm{G}_{\mathrm{k}}\left(\mathrm{x}_{1}, \mathrm{x}_{2}, \cdots, \mathrm{x}_{\mathrm{k}}\right)$ be two arbitrary functions of $m$ and $k$ variables, respectively. If $p>0, q>0$ with $\frac{1}{p}+\frac{1}{q}<1$, then

$$
\begin{gathered}
\int_{a}^{b} H_{l}\left(h_{1}, h_{2}, \cdots, h_{l}\right)\left|F_{m}\left(f_{1}, f_{2}, \cdots, f_{m}\right) G_{k}\left(g_{1}, g_{2}, \cdots, g_{k}\right)\right| d x \\
\leqslant\left(\int_{a}^{b} H_{l}\left(h_{1}, h_{2}, \cdots, h_{l}\right)\left|F_{m}\left(f_{1}, f_{2}, \cdots, f_{m}\right)\right|^{p} d x\right)^{\frac{1}{p}} \\
\quad \times\left(\int_{a}^{b} H_{l}\left(h_{1}, h_{2}, \cdots, h_{l}\right)\left|G_{k}\left(g_{1}, g_{2}, \cdots, g_{k}\right)\right|^{q} d x\right)^{\frac{1}{q}} .
\end{gathered}
$$


Corollary 3.10. $(\mathbb{T}=\mathbb{Z})$ Let $\left\{a_{i 1}, a_{i 2}, \ldots, a_{i m}\right\}_{i=1}^{n},\left\{b_{i 1}, b_{i 2}, \ldots, b_{i k}\right\}_{i=1}^{n}$ and $\left\{c_{i 1}, c_{i 2}, \ldots, c_{i l}\right\}_{i=1}^{n}$ be real numbers for any $\mathrm{m}, \mathrm{k}, \mathrm{l} \in \mathbb{N}$, let $\mathrm{H}_{\mathrm{l}}\left(\mathrm{x}_{1}, \mathrm{x}_{2}, \cdots, \mathrm{x}_{\mathrm{l}}\right)>0$ with $\sum_{i=1}^{n} \mathrm{H}_{l}\left(\mathrm{c}_{i 1}, \mathrm{c}_{\mathrm{i} 2}, \cdots, \mathrm{c}_{i l}\right)=1$, let $\mathrm{F}_{\mathrm{m}}\left(\mathrm{x}_{1}, \mathrm{x}_{2}, \cdots, \mathrm{x}_{\mathrm{m}}\right)$ and $\mathrm{G}_{\mathrm{k}}\left(\mathrm{x}_{1}, \mathrm{x}_{2}, \cdots, \mathrm{x}_{\mathrm{k}}\right)$ be two arbitrary functions of $\mathrm{m}$ and $\mathrm{k}$ variables, respectively. If $\mathrm{p}>0, \mathrm{q}>0$ with $\frac{1}{p}+\frac{1}{q}<1$, then

$$
\begin{aligned}
& \sum_{i=1}^{n} H_{l}\left(c_{i 1}, c_{i 2}, \cdots, c_{i l}\right)\left|F_{m}\left(a_{i 1}, a_{i 2}, \cdots, a_{i m}\right) G_{k}\left(b_{i 1}, b_{i 2}, \cdots, b_{i k}\right)\right| \\
& \leqslant\left(\sum_{i=1}^{n} H_{l}\left(c_{i 1}, c_{i 2}, \cdots, c_{i l}\right)\left|F_{m}\left(a_{i 1}, a_{i 2}, \cdots, a_{i m}\right)\right|^{p}\right)^{\frac{1}{p}} \\
& \quad \times\left(\sum_{i=1}^{n} H_{l}\left(c_{i 1}, c_{i 2}, \cdots, c_{i l}\right)\left|G_{k}\left(b_{i 1}, b_{i 2}, \cdots, b_{i k}\right)\right|^{q}\right)^{\frac{1}{q}} .
\end{aligned}
$$

Theorem 3.11. Let $\mathbb{T}$ be a time scale, $a, b \in \mathbb{T}$ with $a<b$, let $\left\{f_{1 j}(x)\right\}_{j=1}^{k_{1}},\left\{f_{2 j}(x)\right\}_{j=1}^{k_{2}}, \ldots,\left\{f_{m j}(x)\right\}_{j=1}^{k_{m}}$ and $\left\{h_{j}(x)\right\}_{j=1}^{l}$ be continuous real-valued functions on $[\mathrm{a}, \mathrm{b}]_{\mathbb{T}}$ and let $\mathrm{H}_{\mathrm{l}}\left(\mathrm{x}_{1}, \mathrm{x}_{2}, \cdots, \mathrm{x}_{\mathrm{l}}\right)>0, \mathrm{~F}_{\mathrm{k}_{1}}\left(\mathrm{x}_{1}, \mathrm{x}_{2}, \cdots, \mathrm{x}_{\mathrm{k}_{1}}\right)$, $\mathrm{F}_{\mathrm{k}_{2}}\left(\mathrm{x}_{1}, \mathrm{x}_{2}, \cdots, \mathrm{x}_{\mathrm{k}_{2}}\right), \ldots, \mathrm{F}_{\mathrm{k}_{\mathrm{m}}}\left(\mathrm{x}_{1}, \mathrm{x}_{2}, \cdots, \mathrm{x}_{\mathrm{k}_{\mathrm{m}}}\right)$ be $\mathrm{m}+1$ arbitrary functions of $\mathrm{l}, \mathrm{k}_{1}, \mathrm{k}_{2}, \ldots, \mathrm{k}_{\mathrm{m}}$ variables, respectively.

(I) If $1<\lambda_{1}, \lambda_{2}, \cdots, \lambda_{m}<\infty, \sum_{i=1}^{m} \frac{1}{\lambda_{i}}=1$, then

$$
\begin{aligned}
\int_{a}^{b} H_{l}\left(h_{1}, h_{2}, \ldots, h_{l}\right) & \left|\prod_{i=1}^{m} F_{k_{i}}\left(f_{i 1}, f_{i 2}, \cdots, f_{i k_{i}}\right)\right| \diamond_{\alpha} x \\
& \leqslant \prod_{i=1}^{m}\left(\int_{a}^{b} H_{l}\left(h_{1}, h_{2}, \cdots, h_{l}\right)\left|F_{k_{i}}\left(f_{i 1}, f_{i 2}, \cdots, f_{i k_{i}}\right)\right|^{\lambda_{i}} \diamond_{\alpha} x\right)^{\frac{1}{\lambda_{i}}} .
\end{aligned}
$$

(II) If $\lambda_{1}>0, \lambda_{2}, \cdots, \lambda_{m}<\infty$ with $\sum_{i=1}^{m} \frac{1}{\lambda_{i}}=1$, then inequality (3.5) is reversed.

Proof. We need to prove only Case (I). The proof of Case (II) is similar. Inequality (3.5) is trivially true when $F_{k_{i}}\left(f_{i 1}, f_{i 2}, \cdots, f_{i k_{i}}\right)(i=1,2, \cdots, m)$ is identically zero. Let

$$
\prod_{i=1}^{m}\left(\int_{a}^{b} H_{l}\left(h_{1}, h_{2}, \cdots, h_{l}\right)\left|F_{k_{i}}\left(f_{i 1}, f_{i 2}, \cdots, f_{i k_{i}}\right)\right|^{\lambda_{i}} \diamond_{\alpha} x\right)^{\frac{1}{\lambda_{i}}} \neq 0,
$$

and let

$$
\Phi_{i}=\frac{H_{l}^{\frac{1}{\lambda_{i}}}\left(h_{1}, h_{2}, \cdots, h_{l}\right)\left|F_{k_{i}}\left(f_{i 1}, f_{i 2}, \cdots, f_{i k_{i}}\right)\right|}{\left(\int_{a}^{b} H_{l}\left(h_{1}, h_{2}, \cdots, h_{l}\right)\left|F_{k_{i}}\left(f_{i 1}, f_{i 2}, \cdots, f_{i k_{i}}\right)\right|^{\lambda_{i}} \diamond_{\alpha} x\right)^{\frac{1}{\lambda_{i}}}},
$$

where $i=1,2, \cdots, \mathrm{m}$.

By the famous AG inequality

$$
\prod_{i=1}^{m} \Phi_{i} \leqslant \sum_{i=1}^{m} \frac{\Phi_{i}^{\lambda_{i}}}{\lambda_{i}}
$$

(where $1<\lambda_{1}, \lambda_{2}, \cdots, \lambda_{m}<\infty, \sum_{i=1}^{m} \frac{1}{\lambda_{i}}=1$; If $\lambda_{1}>0, \lambda_{2}, \cdots, \lambda_{m}<0, \sum_{i=1}^{m} \frac{1}{\lambda_{i}}=1$, then inequality (3.6) is reversed) we find that

$$
\begin{aligned}
\int_{a}^{b} \prod_{i=1}^{m} \Phi_{i} \diamond_{\alpha} x & \leqslant \int_{a}^{b}\left(\sum_{i=1}^{m} \frac{\Phi_{i}^{\lambda_{i}}}{\lambda_{i}}\right) \diamond_{\alpha} x \\
& =\sum_{i=1}^{m} \frac{1}{\lambda_{i}} \int_{a}^{b} \frac{H_{l}\left(h_{1}, h_{2}, \cdots, h_{l}\right)\left|F_{k_{i}}\left(f_{i 1}, f_{i 2}, \cdots, f_{i k}\right)\right|^{\lambda_{i}}}{\int_{a}^{b} H_{l}\left(h_{1}, h_{2}, \cdots, h_{l}\right)\left|F_{k_{i}}\left(f_{i 1}, f_{i 2}, \cdots, f_{i k}\right)\right|^{\lambda_{i}} \diamond_{\alpha} x} \diamond_{\alpha} x
\end{aligned}
$$




$$
=\sum_{i=1}^{m} \frac{1}{\lambda_{i}}=1 \text {. }
$$

Hence, we get the desired inequality (3.5).

Corollary 3.12. ( $\mathbb{T}=\mathbb{R})$ Let $\left\{\mathrm{f}_{1 j}(x)\right\}_{j=1}^{k_{1}},\left\{f_{2 j}(x)\right\}_{j=1}^{k_{2}}, \ldots,\left\{f_{m j}(x)\right\}_{j=1}^{k_{m}}$ and $\left\{h_{j}(x)\right\}_{j=1}^{l}$ be continuous real-valued functions on $[\mathrm{a}, \mathrm{b}]$ and let $\mathrm{H}_{\mathrm{l}}\left(\mathrm{x}_{1}, \mathrm{x}_{2}, \cdots, \mathrm{x}_{\mathrm{l}}\right)>0, \mathrm{~F}_{\mathrm{k}_{1}}\left(\mathrm{x}_{1}, \mathrm{x}_{2}, \cdots, \mathrm{x}_{\mathrm{k}_{1}}\right), \mathrm{F}_{\mathrm{k}_{2}}\left(\mathrm{x}_{1}, \mathrm{x}_{2}, \cdots, \mathrm{x}_{\mathrm{k}_{2}}\right), \ldots, \mathrm{F}_{\mathrm{k}_{\mathrm{m}}}\left(\mathrm{x}_{1}, \mathrm{x}_{2}, \cdots, \mathrm{x}_{\mathrm{k}_{\mathrm{m}}}\right)$ be $\mathrm{m}+1$ arbitrary functions of $l, \mathrm{k}_{1}, \mathrm{k}_{2}, \ldots, \mathrm{k}_{\mathrm{m}}$ variables, respectively.

(I) If $1<\lambda_{1}, \lambda_{2}, \cdots, \lambda_{m}<\infty, \sum_{i=1}^{m} \frac{1}{\lambda_{i}}=1$, then

$$
\begin{aligned}
& \int_{a}^{b} H_{l}\left(h_{1}, h_{2}, \cdots, h_{l}\right)\left|\prod_{i=1}^{m} F_{k_{i}}\left(f_{i 1}, f_{i 2}, \cdots, f_{i k_{i}}\right)\right| d x \\
& \quad \leqslant \prod_{i=1}^{m}\left(\int_{a}^{b} H_{l}\left(h_{1}, h_{2}, \cdots, h_{l}\right)\left|F_{k_{i}}\left(f_{i 1}, f_{i 2}, \cdots, f_{i k_{i}}\right)\right|^{\lambda_{i}} d x\right)^{\frac{1}{\lambda_{i}}} .
\end{aligned}
$$

(II) If $\lambda_{1}>0, \lambda_{2}, \cdots, \lambda_{m}<\infty$ with $\sum_{i=1}^{m} \frac{1}{\lambda_{i}}=1$, then inequality (3.7) is reversed.

Corollary 3.13. ( $\mathbb{T}=\mathbb{Z}$ ) let $\left\{a_{i 1}^{1}, a_{i 2}^{1}, \ldots, a_{i k_{1}}^{1}\right\}_{i=1}^{n},\left\{a_{i 1}^{2}, a_{i 2}^{2}, \ldots, a_{i k_{2}}^{2}\right\}_{i=1}^{n}, \ldots,\left\{a_{i 1}^{m}, a_{i 2}^{m}, \ldots, a_{i k_{m}}^{m}\right\}_{i=1}^{n}$ and $\left\{c_{i 1}, c_{i 2}, \ldots, c_{i l}\right\}_{i=1}^{n}$ be real numbers for any $k_{1}, k_{2}, \ldots, k_{m}, l \in \mathbb{N}$ and let $H_{l}\left(x_{1}, x_{2}, \cdots, x_{l}\right)>0, F_{k_{1}}\left(x_{1}, x_{2}, \cdots, x_{k_{1}}\right)$, $\mathrm{F}_{\mathrm{k}_{2}}\left(\mathrm{x}_{1}, \mathrm{x}_{2}, \cdots, \mathrm{x}_{\mathrm{k}_{2}}\right), \ldots, \mathrm{F}_{\mathrm{k}_{\mathrm{m}}}\left(\mathrm{x}_{1}, \mathrm{x}_{2}, \ldots, \mathrm{x}_{\mathrm{k}_{\mathrm{m}}}\right)$ be $\mathrm{m}+1$ arbitrary functions of $\mathrm{l}, \mathrm{k}_{1}, \mathrm{k}_{2}, \ldots, \mathrm{k}_{\mathrm{m}}$ variables, respectively.

(I) If $1<\lambda_{1}, \lambda_{2}, \cdots, \lambda_{m}<\infty, \sum_{i=1}^{m} \frac{1}{\lambda_{i}}=1$, then

$$
\begin{aligned}
& \sum_{i=1}^{n} H_{l}\left(c_{i 1}, c_{i 2}, \cdots, c_{i l}\right)\left|\prod_{j=1}^{m} F_{k_{j}}\left(a_{i 1}^{j}, a_{i 2}^{j}, \cdots, a_{i k_{j}}^{j}\right)\right| \\
& \quad \leqslant \prod_{j=1}^{m}\left(\sum_{i=1}^{n} H_{l}\left(c_{i 1}, c_{i 2}, \cdots, c_{i l}\right)\left|F_{k_{j}}\left(a_{i 1}^{j}, a_{i 2}^{j}, \cdots, a_{i k_{j}}^{j}\right)\right|^{\lambda_{j}}\right)^{\frac{1}{\lambda_{j}}} .
\end{aligned}
$$

(II) If $\lambda_{1}>0, \lambda_{2}, \cdots, \lambda_{m}<\infty$ with $\sum_{i=1}^{m} \frac{1}{\lambda_{i}}=1$, then inequality (3.8) is reversed.

Theorem 3.14. Let $\mathbb{T}$ be a time scale, $\mathrm{a}, \mathrm{b} \in \mathbb{T}$ with $\mathrm{a}<\mathrm{b}$, let $\left\{\mathrm{f}_{1 \mathrm{j}}(\mathrm{x})\right\}_{\mathfrak{j}=1}^{\mathrm{k}_{1}},\left\{\mathrm{f}_{2 \mathrm{j}}(x)\right\}_{\mathfrak{j}=1}^{k_{2}}, \ldots,\left\{\mathrm{f}_{\mathrm{mj}}(\mathrm{x})\right\}_{\mathfrak{j}=1}^{k_{\mathrm{m}}}$ and $\left\{h_{j}(x)\right\}_{j=1}^{l}$ be continuous real-valued functions on $[a, b]_{\mathbb{T}}$, let $\mathrm{H}_{l}\left(x_{1}, x_{2}, \cdots, x_{l}\right)>0$ with $\int_{a}^{b} H_{l}\left(h_{1}, h_{2}, \cdots, h_{l}\right) \diamond_{\alpha}$ $x=1$ and let $\mathrm{F}_{\mathrm{k}_{1}}\left(\mathrm{x}_{1}, \mathrm{x}_{2}, \cdots, \mathrm{x}_{\mathrm{k}_{1}}\right), \mathrm{F}_{\mathrm{k}_{2}}\left(\mathrm{x}_{1}, \mathrm{x}_{2}, \cdots, \mathrm{x}_{\mathrm{k}_{2}}\right), \ldots, \mathrm{F}_{\mathrm{k}_{\mathrm{m}}}\left(\mathrm{x}_{1}, \mathrm{x}_{2}, \cdots, \mathrm{x}_{\mathrm{k}_{\mathrm{m}}}\right)$ be $\mathrm{m}$ arbitrary functions of $\mathrm{k}_{1}$, $k_{2}, \ldots, k_{m}$ variables, respectively. If $1<\lambda_{1}, \lambda_{2}, \cdots, \lambda_{m}<\infty$ with $\sum_{i=1}^{m} \frac{1}{\lambda_{i}}<1$, then

$$
\begin{aligned}
& \int_{a}^{b} H_{l}\left(h_{1}, h_{2}, \cdots, h_{l}\right)\left|\prod_{i=1}^{m} F_{k_{i}}\left(f_{i 1}, f_{i 2}, \cdots, f_{i k_{i}}\right)\right| \diamond_{\alpha} x \\
& \leqslant \prod_{i=1}^{m}\left(\int_{a}^{b} H_{l}\left(h_{1}, h_{2}, \cdots, h_{l}\right)\left|F_{k_{i}}\left(f_{i 1}, f_{i 2}, \cdots, f_{i k_{i}}\right)\right|^{\lambda_{i}} \diamond_{\alpha} x\right)^{\frac{1}{\lambda_{i}}} .
\end{aligned}
$$

Proof. Let $\varphi:=\frac{1}{\lambda_{1}}+\frac{1}{\lambda_{2}}+\ldots+\frac{1}{\lambda_{m}}<1, \beta_{i}:=\varphi \lambda_{i}<\lambda_{i}(i=1,2, \ldots, m)$. Then $\frac{1}{\beta_{1}}+\frac{1}{\beta_{2}}+\ldots+\frac{1}{\beta_{m}}=1$. Hence, from Theorem 3.11, Lemma 3.3 we obtain that

$$
\int_{a}^{b} H_{l}\left(h_{1}, h_{2}, \cdots, h_{l}\right)\left|\prod_{i=1}^{m} F_{k_{i}}\left(f_{i 1}, f_{i 2}, \cdots, f_{i k_{i}}\right)\right| \diamond_{\alpha} x
$$




$$
\begin{aligned}
& \leqslant \prod_{i=1}^{m}\left(\int_{a}^{b} H_{l}\left(h_{1}, h_{2}, \cdots, h_{l}\right)\left|F_{k_{i}}\left(f_{i 1}, f_{i 2}, \cdots, f_{i k_{i}}\right)\right|^{\beta_{i}} \diamond_{\alpha} x\right)^{\frac{1}{\beta_{i}}} \\
& \leqslant \prod_{i=1}^{m}\left(\int_{a}^{b} H_{l}\left(h_{1}, h_{2}, \cdots, h_{l}\right)\left|F_{k_{i}}\left(f_{i 1}, f_{i 2}, \cdots, f_{i k_{i}}\right)\right|^{\lambda_{i}} \diamond_{\alpha} x\right)^{\frac{1}{\lambda_{i}}} .
\end{aligned}
$$

The proof of Theorem 3.14 is completed.

Corollary 3.15. $(\mathbb{T}=\mathbb{R})$ Let $\left\{f_{1 j}(x)\right\}_{j=1}^{k_{1}},\left\{f_{2 j}(x)\right\}_{j=1}^{k_{2}}, \ldots,\left\{f_{m j}(x)\right\}_{j=1}^{k_{m}}$ and $\left\{h_{\mathfrak{j}}(x)\right\}_{j=1}^{l}$ be continuous real-valued functions on $[\mathrm{a}, \mathrm{b}]$, let $\mathrm{H}_{\mathrm{l}}\left(\mathrm{x}_{1}, \mathrm{x}_{2}, \cdots, \mathrm{x}_{\mathrm{l}}\right)>0$ with $\int_{\mathrm{a}}^{\mathrm{b}} \mathrm{H}_{\mathrm{l}}\left(\mathrm{h}_{1}, \mathrm{~h}_{2}, \cdots, \mathrm{h}_{\mathrm{l}}\right) \mathrm{dx}=1$, let

$$
F_{k_{1}}\left(x_{1}, x_{2}, \cdots, x_{k_{1}}\right), F_{k_{2}}\left(x_{1}, x_{2}, \cdots, x_{k_{2}}\right), \ldots, F_{k_{m}}\left(x_{1}, x_{2}, \cdots, x_{k_{m}}\right)
$$

be $m$ arbitrary functions of $k_{1}, k_{2}, \ldots, k_{m}$ variables, respectively. If $1<\lambda_{1}, \lambda_{2}, \cdots, \lambda_{m}<\infty$ with $\sum_{i=1}^{m} \frac{1}{\lambda_{i}}<1$, then

$$
\begin{aligned}
\int_{a}^{b} H_{l}\left(h_{1}, h_{2}, \ldots, h_{l}\right)\left|\prod_{i=1}^{m} F_{k_{i}}\left(f_{i 1}, f_{i 2}, \cdots, f_{i k_{i}}\right)\right| d x \\
\leqslant \prod_{i=1}^{m}\left(\int_{a}^{b} H_{l}\left(h_{1}, h_{2}, \cdots, h_{l}\right)\left|F_{k_{i}}\left(f_{i 1}, f_{i 2}, \cdots, f_{i k_{i}}\right)\right|^{\lambda_{i}} d x\right)^{\frac{1}{\lambda_{i}}} .
\end{aligned}
$$

Corollary 3.16. $(\mathbb{T}=\mathbb{Z})$ Let $\left\{a_{i 1}^{1}, a_{i 2}^{1}, \ldots, a_{i k_{1}}^{1}\right\}_{i=1}^{n},\left\{a_{i 1}^{2}, a_{i 2}^{2}, \ldots, a_{i k_{2}}^{2}\right\}_{i=1}^{n}, \ldots,\left\{a_{i 1}^{m}, a_{i 2}^{m}, \ldots, a_{i k_{m}}^{m}\right\}_{i=1}^{n}$ and $\left\{\mathrm{c}_{\mathrm{i} 1}, \mathrm{c}_{\mathrm{i} 2}, \ldots, \mathrm{c}_{\mathrm{il}}\right\}_{i=1}^{\mathrm{n}}$ be real numbers for any $\mathrm{k}_{1}, \mathrm{k}_{2}, \ldots, \mathrm{k}_{\mathrm{m}}, \mathrm{l} \in \mathbb{N}$, let $\mathrm{H}_{\mathrm{l}}\left(\mathrm{x}_{1}, \mathrm{x}_{2}, \ldots, \mathrm{x}_{\mathrm{l}}\right)>0$ with

$$
\sum_{i=1}^{n} H_{l}\left(c_{i 1}, c_{i 2}, \cdots, c_{i l}\right)=1
$$

and let $\mathrm{F}_{\mathrm{k}_{1}}\left(\mathrm{x}_{1}, \mathrm{x}_{2}, \cdots, \mathrm{x}_{\mathrm{k}_{1}}\right), \mathrm{F}_{\mathrm{k}_{2}}\left(\mathrm{x}_{1}, \mathrm{x}_{2}, \cdots, \mathrm{x}_{\mathrm{k}_{2}}\right), \ldots, \mathrm{F}_{\mathrm{k}_{\mathrm{m}}}\left(\mathrm{x}_{1}, \mathrm{x}_{2}, \cdots, \mathrm{x}_{\mathrm{k}_{\mathrm{m}}}\right)$ be $\mathrm{m}$ arbitrary functions of $\mathrm{k}_{1}, \mathrm{k}_{2}, \ldots$, $k_{m}$ variables, respectively. If $1<\lambda_{1}, \lambda_{2}, \cdots, \lambda_{m}<\infty$ with $\sum_{i=1}^{m} \frac{1}{\lambda_{i}}<1$, then

$$
\begin{aligned}
& \sum_{i=1}^{n} H_{l}\left(c_{i 1}, c_{i 2}, \cdots, c_{i l}\right)\left|\prod_{j=1}^{m} F_{k_{j}}\left(a_{i 1}^{j}, a_{i 2}^{j}, \cdots, a_{i k_{j}}^{j}\right)\right| \\
& \quad \leqslant \prod_{j=1}^{m}\left(\sum_{i=1}^{n} H_{l}\left(c_{i 1}, c_{i 2}, \cdots, c_{i l}\right)\left|F_{k_{j}}\left(a_{i 1}^{j}, a_{i 2}^{j}, \cdots, a_{i k_{j}}^{j}\right)\right|^{\lambda_{j}}\right)^{\frac{1}{\lambda_{j}}} .
\end{aligned}
$$

By the same method as in Theorem 3.5, Theorem 3.8, Theorem 3.11 and Theorem 3.14, respectively, we can obtain the following results.

Theorem 3.17. Let $\frac{1}{p}+\frac{1}{q}=\frac{1}{r}$ with $p, q, r \in \mathbb{R}-\{0\}$, let $\mathbb{T}$ be a time scale, $a, b \in \mathbb{T}$ with $a<b$, let $h(x), f(x), g(x):[a, b]_{\mathbb{T}} \rightarrow \mathbb{R}$ be $\diamond_{\alpha}$-integrable functions.

(I) If $\mathrm{p}>0$ and $\mathrm{q}>0$ or $\mathrm{p}>0, \mathrm{q}<0$ and $\mathrm{r}<0$, then

$$
\left(\int_{a}^{b}|h(x)|\left|f^{r}(x) g^{r}(x)\right| \diamond_{\alpha} x\right)^{\frac{1}{r}} \leqslant\left(\int_{a}^{b}|h(x)|\left|f^{p}(x)\right| \diamond_{\alpha} x\right)^{\frac{1}{p}}\left(\int_{a}^{b}\left|h(x) \| g^{q}(x)\right| \diamond_{\alpha} x\right)^{\frac{1}{q}} .
$$

(II) If $\mathrm{p}>0, \mathrm{q}<0$ and $\mathrm{r}>0$ or $\mathrm{p}<0$ and $\mathrm{q}<0$, then

$$
\left(\int_{a}^{b}|h(x)|\left|f^{r}(x) g^{r}(x)\right| \diamond_{\alpha} x\right)^{\frac{1}{r}} \geqslant\left(\int_{a}^{b}|h(x)|\left|f^{p}(x)\right| \diamond_{\alpha} x\right)^{\frac{1}{p}}\left(\int_{a}^{b}|h(x)|\left|g^{q}(x)\right| \diamond_{\alpha} x\right)^{\frac{1}{q}} .
$$


Theorem 3.18. Let $\mathbb{T}$ be a time scale, $\mathrm{a}, \mathrm{b} \in \mathbb{T}$ with $\mathrm{a}<\mathrm{b}$, let $\mathrm{h}(\mathrm{x}), \mathrm{f}(\mathrm{x}), \mathrm{g}(\mathrm{x}):[\mathrm{a}, \mathrm{b}]_{\mathbb{T}} \rightarrow \mathbb{R}$ be $\diamond_{\alpha}$-integrable functions. If $\mathrm{p}>0, \mathrm{q}>0$ with $\frac{1}{\mathrm{p}}+\frac{1}{\mathrm{q}}<1$, then

$$
\int_{a}^{b}|h(x)||f(x) g(x)| \diamond_{\alpha} x \leqslant\left(\int_{a}^{b}|h(x)|\left|f^{p}(x)\right| \diamond_{\alpha} x\right)^{\frac{1}{p}}\left(\int_{a}^{b}\left|h(x) \| g^{q}(x)\right| \diamond_{\alpha} x\right)^{\frac{1}{q}} .
$$

Theorem 3.19. Let $\mathbb{T}$ be a time scale, $\mathrm{a}, \mathrm{b} \in \mathbb{T}$ with $\mathrm{a}<\mathrm{b}$ and let $\mathrm{f}_{\mathfrak{i}}(\mathrm{x})(\mathrm{i}=1,2, \cdots, \mathrm{m}), \mathrm{h}(\mathrm{x}):[\mathrm{a}, \mathrm{b}]_{\mathbb{T}} \rightarrow \mathbb{R}$ be $\diamond_{\alpha}$-integrable functions.

(I) If $1<\lambda_{1}, \lambda_{2}, \cdots, \lambda_{m}<\infty, \sum_{i=1}^{m} \frac{1}{\lambda_{i}}=1$, then

$$
\int_{a}^{b}\left|h(x)\left(\prod_{i=1}^{m} f_{i}(x)\right)\right| \diamond_{\alpha} x \leqslant \prod_{i=1}^{m}\left(\int_{a}^{b}|h(x)|\left|f_{i}(x)\right|^{\lambda_{i}} \diamond_{\alpha} x\right)^{\frac{1}{\lambda_{i}}} .
$$

(II) If $\lambda_{1}>0, \lambda_{2}, \cdots, \lambda_{m}<0$ with $\sum_{i=1}^{m} \frac{1}{\lambda_{i}}=1$, then inequality (3.9) is reversed.

Theorem 3.20. Let $\mathbb{T}$ be a time scale, $\mathrm{a}, \mathrm{b} \in \mathbb{T}$ with $\mathrm{a}<\mathrm{b}$ and let $\mathrm{f}_{\mathfrak{i}}(\mathrm{x})(\mathrm{i}=1,2, \cdots, \mathrm{m}), \mathrm{h}(\mathrm{x}):[\mathrm{a}, \mathrm{b}]_{\mathbb{T}} \rightarrow$ $[0,+\infty)$ be $\diamond_{\alpha}$-integrable functions with $\int_{a}^{b} h(x) \diamond_{\alpha} x=1$. If $1<\lambda_{1}, \lambda_{2}, \cdots, \lambda_{m}<\infty$ with $\frac{1}{\lambda_{1}}+\frac{1}{\lambda_{2}}+\cdots+\frac{1}{\lambda_{m}}<$ 1 , then

$$
\int_{a}^{b} h(x)\left(\prod_{i=1}^{m} f_{i}(x)\right) \diamond_{\alpha} x \leqslant \prod_{i=1}^{m}\left(\int_{a}^{b} h(x) f_{i}^{\lambda_{i}}(x) \diamond_{\alpha} x\right)^{\frac{1}{\lambda_{i}}} .
$$

\section{Applications}

In this section, we firstly present two time scales versions of Minkowski's inequality by using the obtained results.

Theorem 4.1. Let $\mathbb{T}$ be a time scale, $\mathrm{a}, \mathrm{b} \in \mathbb{T}$ with $\mathrm{a}<\mathrm{b}$, let $\mathrm{H}_{\mathrm{l}}\left(\mathrm{x}_{1}, \mathrm{x}_{2}, \cdots, \mathrm{x}_{\mathrm{l}}\right)>0, \mathrm{~F}_{\mathrm{k}_{1}}\left(\mathrm{x}_{1}, \mathrm{x}_{2}, \cdots, \mathrm{x}_{\mathrm{k}_{1}}\right)$, $\mathrm{F}_{\mathrm{k}_{2}}\left(\mathrm{x}_{1}, \mathrm{x}_{2}, \cdots, \mathrm{x}_{\mathrm{k}_{2}}\right), \ldots, \mathrm{F}_{\mathrm{k}_{\mathrm{m}}}\left(\mathrm{x}_{1}, \mathrm{x}_{2}, \cdots, \mathrm{x}_{\mathrm{k}_{\mathrm{m}}}\right)$ be $\mathrm{m}+1$ arbitrary nonnegative real-valued functions of $\mathrm{l}, \mathrm{k}_{1}, \mathrm{k}_{2}$, $\ldots, \mathrm{k}_{\mathrm{m}}$ variables, respectively and let $\left\{\mathrm{f}_{1 j}(\mathrm{x})\right\}_{j=1}^{k_{1}},\left\{\mathrm{f}_{2 j}(\mathrm{x})\right\}_{\mathfrak{j}=1}^{\mathrm{k}_{2}}, \ldots,\left\{\mathrm{f}_{\mathrm{mj}}(\mathrm{x})\right\}_{\mathfrak{j}=1}^{\mathrm{k}_{\mathrm{m}}}$ and $\left\{\mathrm{h}_{\mathfrak{j}}(\mathrm{x})\right\}_{\mathfrak{j}=1}^{\mathrm{l}}$ be continuous realvalued functions on $[a, b]_{\mathbb{T}}$.

(I) If $\mathrm{p}>1$, then

$$
\begin{aligned}
& {\left[\int_{a}^{b} H_{l}\left(h_{1}, h_{2}, \cdots, h_{l}\right)\left(\sum_{i=1}^{m}\left|F_{k_{i}}\left(f_{i 1}, f_{i 2}, \cdots, f_{i k_{i}}\right)\right|\right)^{p} \diamond_{\alpha} x\right]^{\frac{1}{p}}} \\
& \quad \leqslant \sum_{i=1}^{m}\left(\int_{a}^{b} H_{l}\left(h_{1}, h_{2}, \cdots, h_{l}\right)\left|F_{k_{i}}\left(f_{i 1}, f_{i 2}, \cdots, f_{i k_{i}}\right)\right|^{p} \diamond_{\alpha} x\right)^{\frac{1}{p}} .
\end{aligned}
$$

(II) If $0<p<1$, then

$$
\begin{aligned}
& {\left[\int_{a}^{b} H_{l}\left(h_{1}, h_{2}, \cdots, h_{l}\right)\left(\sum_{i=1}^{m}\left|F_{k_{i}}\left(f_{i 1}, f_{i 2}, \cdots, f_{i k_{i}}\right)\right|\right)^{p} \diamond_{\alpha} x\right]^{\frac{1}{p}}} \\
& \quad \geqslant \sum_{i=1}^{m}\left(\int_{a}^{b} H_{l}\left(h_{1}, h_{2}, \cdots, h_{l}\right)\left|F_{k_{i}}\left(f_{i 1}, f_{i 2}, \cdots, f_{i k_{i}}\right)\right|^{p} \diamond_{\alpha} x\right)^{\frac{1}{p}} .
\end{aligned}
$$

Proof. We only consider the case (I). For $\mathrm{p}>1$, from Jenson's inequality, we have

$$
\left(\sum_{i=1}^{m} F_{k_{i}}\left(f_{i 1}(x), f_{i 2}(x), \cdots, f_{i k_{i}}(x)\right)\right)^{p} \geqslant \sum_{i=1}^{m} F_{k_{i}}^{p}\left(f_{i 1}(x), f_{i 2}(x), \cdots, f_{i k_{i}}(x)\right) .
$$

Denote $\Phi(x)=\sum_{i=1}^{m} F_{k_{i}}\left(f_{i 1}(x), f_{i 2}(x), \cdots, f_{i k_{i}}(x)\right)$. Without loss of generality, we may assume that $\int_{a}^{b} h(x) \Phi^{p}(x) \diamond_{\alpha} x>0$. From Theorem 3.5, for $r=1, p>0, q>0$ and $\frac{1}{p}+\frac{1}{q}=1$, we obtain that 


$$
\begin{aligned}
& \int_{a}^{b}\left|F_{k_{i}}\left(f_{i 1}, f_{i 2}, \cdots, f_{i k_{i}}\right) H_{l}\left(h_{1}, h_{2}, \cdots, h_{l}\right) \Phi^{p-1}(x)\right| \diamond_{\alpha} x \\
& \leqslant\left(\int_{a}^{b}\left|H_{l}\left(h_{1}, h_{2}, \cdots, h_{l}\right)\right|\left|F_{k_{i}}\left(f_{i 1}, f_{i 2}, \cdots, f_{i k_{i}}\right)\right|^{p} \diamond_{\alpha} x\right)^{\frac{1}{p}} \\
& \quad \times\left(\int_{a}^{b}\left|H_{l}\left(h_{1}, h_{2}, \cdots, h_{l}\right)\right||\Phi(x)|^{(p-1) q} \diamond_{\alpha} x\right)^{\frac{1}{q}} \\
& =\left(\int_{a}^{b}\left|H_{l}\left(h_{1}, h_{2}, \cdots, h_{l}\right) \| F_{k_{i}}\left(f_{i 1}, f_{i 2}, \cdots, f_{i k_{i}}\right)\right|^{p} \diamond_{\alpha} x\right)^{\frac{1}{p}} \\
& \quad \times\left(\int_{a}^{b}\left|H_{l}\left(h_{1}, h_{2}, \cdots, h_{l}\right) \| \Phi(x)\right|^{p} \diamond_{\alpha} x\right)^{\frac{1}{q}} .
\end{aligned}
$$

Hence, from inequality (4.3), we get

$$
\begin{aligned}
\int_{a}^{b} \mid & H_{l}\left(h_{1}, h_{2}, \cdots, h_{l}\right) \Phi^{p}(x) \mid \diamond_{\alpha} x \\
= & \int_{a}^{b}\left|H_{l}\left(h_{1}, h_{2}, \cdots, h_{l}\right) \Phi(x) \Phi^{p-1}(x)\right| \diamond_{\alpha} x \\
= & \int_{a}^{b}\left|H_{l}\left(h_{1}, h_{2}, \cdots, h_{l}\right) F_{k_{1}}\left(f_{11}, f_{12}, \cdots, f_{1 k_{1}}\right) \Phi^{p-1}(x)\right| \diamond_{\alpha} x \\
& +\ldots+\int_{a}^{b}\left|H_{l}\left(h_{1}, h_{2}, \cdots, h_{l}\right) F_{k_{m}}\left(f_{m 1}, f_{m 2}, \cdots, f_{m k_{m}}\right) \Phi^{p-1}(x)\right| \diamond_{\alpha} x \\
\leqslant & \left(\int_{a}^{b}\left|H_{l}\left(h_{1}, h_{2}, \cdots, h_{l}\right)\right||\Phi(x)|^{p} \diamond_{\alpha} x\right)^{\frac{1}{q}} \\
& \times\left[\sum_{i=1}^{m}\left(\int_{a}^{b}\left|H_{l}\left(h_{1}, h_{2}, \cdots, h_{l}\right)\right|\left|F_{k_{i}}\left(f_{i 1}, f_{i 2}, \cdots, f_{i k_{i}}\right)\right|^{p} \diamond_{\alpha} x\right)^{\frac{1}{p}}\right] .
\end{aligned}
$$

Thus, we obtain the desired inequality (4.1).

Remark 4.2. If $p>1, m=2$, then inequality (4.1) reduces to inequality (2.5) obtained by Yang [26]. If $0<p<1, m=2$, then inequality (4.2) reduces to inequality (2.4) obtained by Chen and Chen [6].

Theorem 4.3. Let $\mathbb{T}$ be a time scale, $\mathrm{a}, \mathrm{b} \in \mathbb{T}$ with $\mathrm{a}<\mathrm{b}$ and let $\mathrm{f}_{\mathfrak{i}}(\mathrm{x})(\mathrm{i}=1,2, \cdots, \mathrm{m}), \mathrm{h}(\mathrm{x}):[\mathrm{a}, \mathrm{b}]_{\mathbb{T}} \rightarrow \mathbb{R}$ be $\diamond_{\alpha}$-integrable functions.

(I) If $\mathrm{p}>1$, then

$$
\left[\int_{a}^{b}\left|h(x)\left(\sum_{i=1}^{m} f_{i}(x)\right)^{p}\right| \diamond_{\alpha} x\right]^{\frac{1}{p}} \leqslant \sum_{i=1}^{m}\left(\int_{a}^{b}|h(x)|\left|f_{i}(x)\right|^{p} \diamond_{\alpha} x\right)^{\frac{1}{p}} .
$$

(II) If $0<p<1$, then

$$
\left[\int_{a}^{b}\left|h(x)\left(\sum_{i=1}^{m} f_{i}(x)\right)^{p}\right| \diamond_{\alpha} x\right]^{\frac{1}{p}} \geqslant \sum_{i=1}^{m}\left(\int_{a}^{b}|h(x)|\left|f_{i}(x)\right|^{p} \diamond_{\alpha} x\right)^{\frac{1}{p}} .
$$

Remark 4.4. If $p>1, m=2$, then inequality (4.4) reduces to inequality (3.7) obtained by Özkan et al. [15]. If $0<p<1, m=2$, then inequality (4.5) is the reversed version of inequality (3.7) obtained by Özkan et al. [15]. 
Secondly, we shall establish two time scales versions of Beckenbach-Dresher's inequality [7] via diamond- $\alpha$ integral.

Theorem 4.5. Let $\mathbb{T}$ be a time scale, $a, b \in \mathbb{T}$ with $a<b$, let $\left\{f_{1 j}(x)\right\}_{j=1}^{k_{1}},\left\{f_{2 j}(x)\right\}_{j=1}^{k_{2}}, \ldots,\left\{f_{m j}(x)\right\}_{j=1}^{k_{m}}$ and $\left\{h_{j}(x)\right\}_{j=1}^{l}$ be continuous real-valued functions on $[a, b]_{\mathbb{T}}$, let $F_{k_{1}}\left(x_{1}, x_{2}, \cdots, x_{k_{1}}\right), F_{k_{2}}\left(x_{1}, x_{2}, \cdots, x_{k_{2}}\right), \ldots$, $\mathrm{F}_{\mathrm{k}_{\mathrm{m}}}\left(\mathrm{x}_{1}, \mathrm{x}_{2}, \cdots, \mathrm{x}_{\mathrm{k}_{\mathrm{m}}}\right)$ be $\mathrm{m}$ arbitrary nonnegative real-valued functions of $\mathrm{k}_{1}, \mathrm{k}_{2}, \ldots, \mathrm{k}_{\mathrm{m}}$ variables, respectively, and let $\mathrm{H}_{\mathrm{l}}\left(\mathrm{x}_{1}, \mathrm{x}_{2}, \cdots, \mathrm{x}_{\mathrm{l}}\right)>0$ with $\int_{\mathrm{a}}^{\mathrm{b}} \mathrm{H}_{\mathrm{l}}^{\mathrm{r}}\left(\mathrm{h}_{1}, \mathrm{~h}_{2}, \cdots, \mathrm{h}_{\mathrm{l}}\right) \diamond_{\alpha} \mathrm{x}>0$.

(I) If $p \geqslant 1 \geqslant r \geqslant 0$, then

$$
\begin{gathered}
\left(\frac{\int_{a}^{b} H_{l}\left(h_{1}, h_{2}, \cdots, h_{l}\right)\left(\sum_{i=1}^{m} F_{k_{i}}\left(f_{i 1}, f_{i 2}, \cdots, f_{i k_{i}}\right)\right)^{p} \diamond_{\alpha} x}{\int_{a}^{b} H_{l}\left(h_{1}, h_{2}, \cdots, h_{l}\right)\left(\sum_{i=1}^{m} F_{k_{i}}\left(f_{i 1}, f_{i 2}, \cdots, f_{i k_{i}}\right)\right)^{r} \diamond_{\alpha} x}\right)^{\frac{1}{p-r}} \\
\quad \leqslant \sum_{i=1}^{m}\left(\frac{\int_{a}^{b} H_{l}\left(h_{1}, h_{2}, \cdots, h_{l}\right) F_{k_{i}}^{p}\left(f_{i 1}, f_{i 2}, \cdots, f_{i k_{i}}\right) \diamond_{\alpha} x}{\int_{a}^{b} H_{l}\left(h_{1}, h_{2}, \cdots, h_{l}\right) F_{k_{i}}^{r}\left(f_{i 1}, f_{i 2}, \cdots, f_{i k_{i}}\right) \diamond_{\alpha} x}\right)^{\frac{1}{p-r}} .
\end{gathered}
$$

(II) If $0<p<r$ or $r<p<0$, then inequality (4.6) is reversed.

Proof. Case (I). Let $p \geqslant 1>r>0$. Write $\gamma=\frac{r}{p-r}>0$,

$$
\zeta_{i}=\left(\int_{a}^{b} H_{l}\left(h_{1}, h_{2}, \cdots, h_{l}\right) F_{k_{i}}^{p}\left(f_{i 1}, f_{i 2}, \cdots, f_{i k_{i}}\right) \diamond_{\alpha} x\right)^{\frac{1}{p}},
$$

and

$$
\xi_{i}=\left(\int_{a}^{b} H_{l}\left(h_{1}, h_{2}, \cdots, h_{l}\right) F_{k_{i}}^{r}\left(f_{i 1}, f_{i 2}, \cdots, f_{i k_{i}}\right) \diamond_{\alpha} x\right)^{\frac{1}{r}} .
$$

Then $\gamma+1=\frac{p}{p-r}$. By Lemma 3.2, we have

$$
\begin{aligned}
\frac{\left(\sum_{i=1}^{m} \zeta_{i}\right)^{\gamma+1}}{\left(\sum_{i=1}^{m} \xi_{i}\right)^{\gamma}} & =\frac{\left[\sum_{i=1}^{m}\left(\int_{a}^{b} H_{l}\left(h_{1}, h_{2}, \cdots, h_{l}\right) F_{k_{i}}^{p}\left(f_{i 1}, f_{i 2}, \cdots, f_{i k_{i}}\right) \diamond_{\alpha} x\right)^{\frac{1}{p}}\right]^{\frac{p}{p-r}}}{\left[\sum_{i=1}^{m}\left(\int_{a}^{b} H_{l}\left(h_{1}, h_{2}, \cdots, h_{l}\right) F_{k_{i}}^{r}\left(f_{i 1}, f_{i 2}, \cdots, f_{i k_{i}}\right) \diamond_{\alpha} x\right)^{\frac{1}{r}}\right]^{\frac{r}{p-r}}} \\
& \leqslant \sum_{i=1}^{m}\left(\frac{\left[\left(\int_{a}^{b} H_{l}\left(h_{1}, h_{2}, \cdots, h_{l}\right) F_{k_{i}}^{p}\left(f_{i 1}, f_{i 2}, \cdots, f_{i k_{i}}\right) \diamond_{\alpha} x\right)^{\frac{1}{p}}\right]^{\frac{p}{p-r}}}{\left[\left(\int_{a}^{b} H_{l}\left(h_{1}, h_{2}, \cdots, h_{l}\right) F_{k_{i}}^{r}\left(f_{i 1}, f_{i 2}, \cdots, f_{i k_{i}}\right) \diamond_{\alpha} x\right)^{\frac{1}{r}}\right]^{\frac{r}{p-r}}}\right) \\
& =\sum_{i=1}^{m}\left(\frac{\int_{a}^{b} H_{l}\left(h_{1}, h_{2}, \cdots, h_{l}\right) F_{k_{i}}^{p}\left(f_{i 1}, f_{i 2}, \cdots, f_{i k_{i}}\right) \diamond_{\alpha} x}{\int_{a}^{b} H_{l}\left(h_{1}, h_{2}, \cdots, h_{l}\right) F_{k_{i}}^{r}\left(f_{i 1}, f_{i 2}, \cdots, f_{i k_{i}}\right) \diamond_{\alpha} x}\right)^{\frac{1}{p-r}} .
\end{aligned}
$$

On the other hand, from $p \geqslant 1>r>0$ and Theorem 4.1, we have

$$
\begin{aligned}
& {\left[\sum_{i=1}^{m}\left(\int_{a}^{b} H_{l}\left(h_{1}, h_{2}, \cdots, h_{l}\right) F_{k_{i}}^{p}\left(f_{i 1}, f_{i 2}, \cdots, f_{i k_{i}}\right) \diamond_{\alpha} x\right)^{\frac{1}{p}}\right]^{p}} \\
& \quad \geqslant \int_{a}^{b} H_{l}\left(h_{1}, h_{2}, \cdots, h_{l}\right)\left(\sum_{i=1}^{m} F_{k_{i}}\left(f_{i 1}, f_{i 2}, \cdots, f_{i k_{i}}\right)\right)^{p} \diamond_{\alpha} x,
\end{aligned}
$$

and

$$
\begin{aligned}
& {\left[\sum_{i=1}^{m}\left(\int_{a}^{b} H_{l}\left(h_{1}, h_{2}, \cdots, h_{l}\right) F_{k_{i}}^{r}\left(f_{i 1}, f_{i 2}, \cdots, f_{i k_{i}}\right) \diamond_{\alpha} x\right)^{\frac{1}{r}}\right]^{r}} \\
& \quad \leqslant \int_{a}^{b} H_{l}\left(h_{1}, h_{2}, \cdots, h_{l}\right)\left(\sum_{i=1}^{m} F_{k_{i}}\left(f_{i 1}, f_{i 2}, \cdots, f_{i k_{i}}\right)\right)^{r} \diamond_{\alpha} x .
\end{aligned}
$$


Combining inequalities (4.7), (4.8) and (4.9) yields inequality (4.6) immediately. Clearly, the desired inequality (4.6) also holds for $p=r$ or $r=0$. This completes the proof of Case (I).

Similarly, we can prove the Case (II). The proof of Theorem 4.5 is completed.

Similar to the proof of Theorem 4.5 but using Theorem 4.3 in place of Theorem 4.1 , we immediately obtain the following result.

Theorem 4.6. Let $\mathbb{T}$ be a time scale, $\mathrm{a}, \mathrm{b} \in \mathbb{T}$ with $\mathrm{a}<\mathrm{b}$, let $\mathrm{f}_{\mathfrak{i}}(\mathrm{x})(\mathrm{i}=1,2, \cdots, \mathrm{m}), \mathrm{h}(\mathrm{x}):[\mathrm{a}, \mathrm{b}]_{\mathbb{T}} \rightarrow[0, \infty)$ be $\diamond_{\alpha}$-integrable functions and let $\int_{a}^{b} h(x) f_{i}^{r}(x) \diamond_{\alpha} x>0(i=1,2, \ldots, m)$.

(I) If $p \geqslant 1 \geqslant r \geqslant 0$, then

$$
\left(\frac{\int_{a}^{b} h(x)\left(\sum_{i=1}^{m} f_{i}(x)\right)^{p} \diamond_{\alpha} x}{\int_{a}^{b} h(x)\left(\sum_{i=1}^{m} f_{i}(x)\right)^{r} \diamond_{\alpha} x}\right)^{\frac{1}{p-r}} \leqslant \sum_{i=1}^{m}\left(\frac{\int_{a}^{b} h(x)\left(f_{i}(x)\right)^{p} \diamond_{\alpha} x}{\int_{a}^{b} h(x)\left(f_{i}(x)\right)^{r} \diamond_{\alpha} x}\right)^{\frac{1}{p-r}} .
$$

(II) If $0<p<r$ or $r<p<0$, then inequality (4.10) is reversed.

\section{Acknowledgment}

The authors would like to express their sincere thanks to the anonymous referees for their great efforts to improve this paper.

This work was supported by the NNSF of China (No. 61073121), the Application Basic Research Plan Key Basic Research Project of Hebei Province of China (No. 16964213D), the Fundamental Research Funds for the Central Universities (No. 2015ZD29) and the Higher School Science Research Funds of Hebei Province of China (No. Z2015137).

\section{References}

[1] S. Abramovich, J. Pečarić, S. Varošanec, Sharpening Hölder's and Popoviciu's inequalities via functionals, Rocky Mountain J. Math., 34 (2004), 793-810. 1

[2] S. Abramovich, J. Pečarić, S. Varošanec, Continuous sharpening of Hölder's and Minkowski's inequalities, Math. Inequal. Appl., 8 (2005), 179-190. 1

[3] R. Agarwal, M. Bohner, A. Peterson, Inequalities on time scales: a survey, Math. Inequal. Appl., 4 (2001), 535-557. 2

[4] M. Bohner, A. Peterson, Dynamic equations on time scales, An introduction with applications. Birkhäuser Boston, Inc., Boston, MA, (2001). 2.6, 2.7

[5] M. Bohner, A. Peterson, Eds., Advances in dynamic equations on time scales, Birkhäuser, Boston, Mass, USA, (2003). 2.1, 2.2

[6] G.-S. Chen, Z. Chen, A functional generalization of the reverse Hölder integral inequality on time scales, Math. Comput. Modelling, 54 (2011), 2939-2942. 1, 4.2

[7] G. H. Hardy, J. E. Littlewood, G. Pólya, Inequalities, 2d ed, Cambridge, at the University Press, (1952). 3.2, 3, 3, 4

[8] S. Hilger, Ein maßkettenkalkül mit anwendung auf zentrumsmannigfaltigkeiten, Ph.D. thesis, Universität Würzburg, Würzburg, Germany, (1988). 2

[9] S. Hilger, Analysis on measure chains - a unified approach to continuous and discrete calculus, Results Math., 18 (1990), 18-56. 2.3, 2.4, 2.5, 2

[10] S. Hilger, Differential and difference calculus-unified, Proceedings of the Second World Congress of Nonlinear Analysts, Part 5, Athens, (1996), Nonlinear Anal., 30 (1997), 2683-2694. 2

[11] J. Kuang, Applied inequalities, Shandong Science Press, Jinan, (2003). 1

[12] B.-D. Liu, Random fuzzy dependent-chance programming and its hybrid intelligent algorithm, Inform. Sci., 141 (2002), 259-271. 1

[13] J. Matkowski, A converse of the Hölder inequality theorem, Math. Inequal. Appl., 12 (2009), 21-32. 1

[14] D. S. Mitrinović, Analytic inequalities, In cooperation with P. M. Vasić, Die Grundlehren der mathematischen Wissenschaften, Band 165 Springer-Verlag, New York-Berlin, (1970). 1

[15] U. M. Özkan, M. Z. Sarikaya, H. Yildirim, Extensions of certain integral inequalities on time scales, Appl. Math. Lett., 21 (2008), 993-1000. 1, 4.4

[16] J. W. Rogers Jr., Q. Sheng, Notes on the diamond- $\alpha$ dynamic derivative on time scales, J. Math. Anal. Appl., 326 (2007), 228-241. 2 
[17] Q. Sheng, M. Fadag, J. Henderson, J. M. Davis, An exploration of combined dynamic derivatives on time scales and their applications, Nonlinear Anal. Real World Appl., 7 (2006), 395-413. 2.8, 2.9, 2.10, 2.11, 2.12, 2.13, 3.1

[18] J.-F. Tian, New property of a generalized Hölder's inequality and its applications, Inform. Sci., 288 (2014), 45-54. 1

[19] J.-F. Tian, Properties of generalized Hölder's inequalities, J. Math. Inequal., 9 (2015), 473-480. 1

[20] J.-F. Tian, M.-H. Ha, Some new properties of generalized Hölder's inequalities, J. Nonlinear Sci. Appl., 10 (2016), $3638-$ 3646. 1

[21] J.-F. Tian, M.-H. Ha, Properties of generalized sharp Hölder's inequalities, J. Math. Inequal., 11 (2017), 511-525. 1

[22] F.-H. Wong, C.-C. Yeh, W.-C. Lian, An extension of Jensen's inequality on time scales, Adv. Dyn. Syst. Appl., 1 (2006), 113-120. 2

[23] F.-H. Wong, C.-C. Yeh, S.-L. Yu, C.-H. Hong, Young's inequality and related results on time scales, Appl. Math. Lett., 18 (2005), 983-988. 1

[24] S.-H. Wu, Generalization of a sharp Hölder's inequality and its application, J. Math. Anal. Appl., 332 (2007), 741-750. 1

[25] S.-H. Wu, A new sharpened and generalized version of Hölder's inequality and its applications, Appl. Math. Comput., 197 (2008), 708-714. 1

[26] W.-G. Yang, A functional generalization of diamond- $\alpha$ integral Hölder's inequality on time scales, Appl. Math. Lett., 23 (2010), 1208-1212. 1, 4.2 